\title{
The deubiquitinase (DUB) USP13 promotes Mcl-1 stabilisation in cervical cancer
}

\author{
Ethan L. Morgan (1) 1,2,3 Molly R. Patterson ${ }^{1,2}$ - Diego Barba-Moreno ${ }^{1,2} \cdot$ James A. Scarth $^{1,2}$ - Adam Wilson ${ }^{1,2}$. \\ Andrew Macdonald $\oplus^{1,2}$
}

Received: 25 July 2020 / Revised: 13 January 2021 / Accepted: 25 January 2021 / Published online: 24 February 2021

(c) The Author(s) 2021. This article is published with open access

\begin{abstract}
Protein ubiquitination is a critical regulator of cellular homeostasis. Aberrations in the addition or removal of ubiquitin can result in the development of cancer and key components of the ubiquitination machinery serve as oncogenes or tumour suppressors. An emerging target in the development of cancer therapeutics are the deubiquitinase (DUB) enzymes that remove ubiquitin from protein substrates. Whether this class of enzyme plays a role in cervical cancer has not been fully explored. By interrogating the cervical cancer data from the TCGA consortium, we noted that the DUB USP13 is amplified in $\sim 15 \%$ of cervical cancer cases. We confirmed that USP13 expression was increased in cervical cancer cell lines, cytology samples from patients with cervical disease and in cervical cancer tissue. Depletion of USP13 inhibited cervical cancer cell proliferation. Mechanistically, USP13 bound to, deubiquitinated and stabilised Mcl-1, a pivotal member of the anti-apoptotic BCL-2 family. Furthermore, reduced Mcl-1 expression partially contributed to the observed proliferative defect in USP13 depleted cells. Importantly, the expression of USP13 and Mcl-1 proteins correlated in cervical cancer tissue. Finally, we demonstrated that depletion of USP13 expression or inhibition of USP13 enzymatic activity increased the sensitivity of cervical cancer cells to the BH3 mimetic inhibitor ABT-263. Together, our data demonstrates that USP13 is a potential oncogene in cervical cancer that functions to stabilise the pro-survival protein Mcl-1, offering a potential therapeutic target for these cancers.
\end{abstract}

\section{Introduction}

Protein ubiquitination is a critical post-translation modification that is essential for the regulation of cellular

Supplementary information The online version contains supplementary material available at https://doi.org/10.1038/s41388021-01679-8.

Ethan L. Morgan

ethan.morgan@nih.gov

$\triangle$ Andrew Macdonald

a.macdonald@leeds.ac.uk

1 School of Molecular and Cellular Biology, Faculty of Biological Sciences, University of Leeds, Leeds, West Yorkshir, UK

2 Astbury Centre for Structural Molecular Biology, University of Leeds, Leeds, West Yorkshire, UK

3 Present address: Tumor Biology Section, Head and Neck Surgery Branch, National Institute of Deafness and Other Communication Disorders, National Institute of Health, Bethesda, MD, USA homeostasis [1]. The process of ubiquitination occurs through a stepwise enzymatic cascade comprising three classes of enzymes: E1 ubiquitin-activating enzymes, E2 ubiquitin-conjugating enzymes and E3 ubiquitin ligases. The functional outcome of protein ubiquitination is dependent on the type of modification (monoubiquitin or polyubiquitin) and the linkage type within the ubiquitin chain [2]. This diversity of potential ubiquitin signals is able to regulate a wide range of cellular processes. For example, K48 or K11 polyubiquitin chains are primarily responsible for promoting protein degradation, whereas chains linked by M1 or K63 polyubiquitin direct the assembly of protein complexes to regulate signal transduction [3]. Consequently, the deregulation of protein ubiquitination can result in the promotion of diseases including cancer. For example, defects in ubiquitin-mediated proteasomal degradation can result in either the enhanced degradation of tumour suppressor proteins, or in the stabilisation of oncogenes [4].

Ubiquitination is a highly dynamic and reversible posttranslational modification, and deubiquitinase enzymes (DUBs) readily cleave ubiquitin from its protein substrates 
[5]. To date, over 100 DUBs have been identified in the human genome that are classified into seven families on the basis of the catalytic mechanism and structural similarity; members of the ubiquitin C-terminal hydrolases (UCHs), ubiquitin-specific proteases (USPs), ovarian tumour proteases (OTUs), the Machado-Josephin domain superfamily (MJD), the MINDY family and the ZUFSP family function as cysteine proteases, whereas JAB1/MPN/MOV34 metalloenzymes (JAMMs) are zinc-dependent metalloproteases [5]. In the last 10 years, DUBs have been shown to regulate the actions of proteins involved in cancer progression, including the tumour suppressors p53 and PTEN, and the oncogenes c-Myc and EGFR [6-9]. Therefore, the pharmacological targeting of DUBs offers a potential therapeutic opportunity for treating cancers [4]. For example, FT671 is a highly specific, non-covalent small molecule inhibitor of USP7, a DUB that regulates MDM2 levels, a critical negative regulator of the p53 tumour suppressor $[10,11]$. FT671 efficiently destabilised MDM2 in Multiple Myeloid MM.1s cells, resulting in increased p53 expression and inhibiting tumour growth in murine xenografts [11].

Cervical cancer is the fourth most common cancer in women, causing significant morbidity and mortality worldwide [12]. Persistent infection with human papillomavirus (HPV) is the underlying cause of almost all cervical cancers, with the high-risk (HR) HPV16 and HPV18 types being the two most prevalent HPV types responsible [13]. HPV-induced transformation is primarily driven by the E6 and E7 viral oncogenes [14, 15], and this is achieved through the extensive manipulation of host cell signalling networks [16-21]. Critically, E6 and E7 promote the proteasomal degradation of the p53 and pRb tumour suppressors, by co-opting the E3 ligases E6-associated protein (E6-AP) and a Cullin-2 ubiquitin ligase complex [22-24]. In addition, the minor HPV oncoprotein E5 can enhance EGFR signalling [25-28], in part by disrupting the c-Cbl/ EGFR complex, thereby decreasing c-Cbl-mediated ubiquitination and degradation of the EGFR [29]. Several DUBs are also targeted during the HPV life cycle and in HPV-associated cancers [30-33]. These data suggest that the deregulation of protein ubiquitination plays a key role in HPV associated cancers. To identify novel DUBs involved in cervical cancer progression, we interrogated the cervical cancer data from the TCGA consortium. We identified that USP13 is amplified in about $15 \%$ of cervical cancer cases. We further demonstrated that USP13 was required for the proliferation of HPV positive cervical cancer cells, at least in part via the deubiquitination and stabilisation of the prosurvival protein, Mcl-1. Crucially, we demonstrated that pharmacological inhibition of USP13 sensitises HPV positive cervical cancer cells to $\mathrm{BH} 3$ mimetic inhibitors, suggesting that the targeting of USP13 may have therapeutic benefit in these cancers.

\section{Results}

\section{Increased USP13 expression in pre-malignant cervical disease and cervical cancer}

To investigate whether DUBs contribute to transformation in cervical cancer, we analysed the cervical cancer dataset from The Cancer Genome Atlas (TCGA) [34]. By examining the copy number alterations (CNAs) of DUB genes in the human genome using a previously curated gene list ([35]; Supplementary Fig. 1) we observed amplification of USP13 in approximately $15 \%$ of cervical cancers, which was also seen in a number of other squamous carcinomas (Supplementary Fig. 1 and Fig. 1A). Importantly, USP13 copy number positively correlated with USP13 mRNA expression in cervical cancer $(R=0.350)$ (Fig. 1B). Next, we examined the expression of USP13 in a panel of cervical cancer cell lines. When compared to primary normal human keratinocytes (NHKs), USP13 mRNA expression was higher in HPV positive (HPV + ), but not HPV negative (HPV-) cervical cancer cells (Fig. 1C). In contrast, USP13 protein levels were increased in both HPV + and HPVcervical cancer cells compared to NHKs, when analysed by western blot (Fig. 1D). To confirm the increased USP13 protein expression in cervical cancer, we performed immunohistochemistry (IHC) on a cervical cancer tissue microarray (TMA). In line with the data from cell lines, USP13 protein expression was significantly higher in the cervical cancer tissue (Fig. 1E). The development of cervical cancer occurs over many years, through the accumulation of pre-malignant alteration of the squamous epithelia collectively known as cervical intraepithelial neoplasia (CIN); CIN1 represents a transient HPV infection with mild dysplasia, while CIN3 represents severe dysplasia which may develop into cervical cancer [36]. To investigate if USP13 expression may contribute to the development of cervical cancer, we analysed USP13 mRNA expression in cervical cytology samples from a cohort of HPV16+ patients. Samples from healthy, HPV-patients were used as controls. USP13 mRNA expression and the levels of USP13 protein both increased during progression through CIN1 to CIN3 (Fig. 1F, G). In validation of our data in cervical cancer cell lines and cervical cancer tissue, USP13 mRNA expression was also significantly upregulated in several published microarray databases (Supplementary Fig. 2), suggesting that increased USP13 expression is a common occurrence in cervical cancer.

\section{HR-HPV oncogenes do not modulate USP13 expression in cervical cancer}

Our cell line data showed higher USP13 expression in HPV + cervical cancer cells compared to normal control cells 
A USP13 Copy Number Alterations

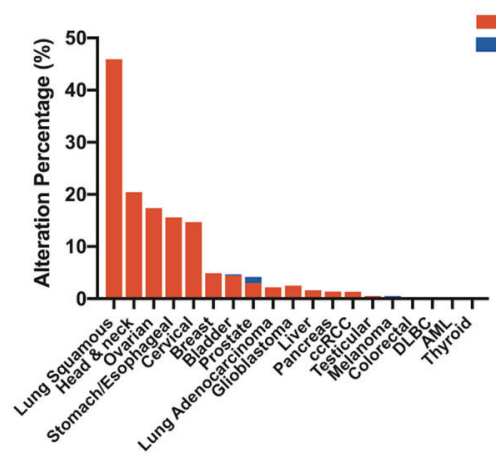

C

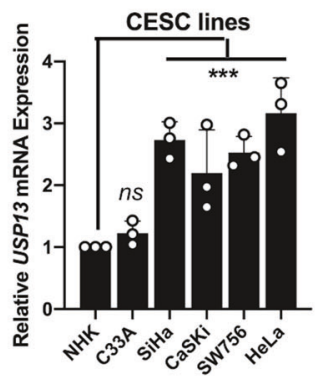

E

Non-cancer Cervical cancer

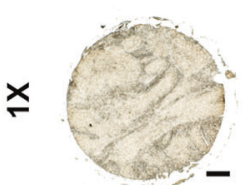

×
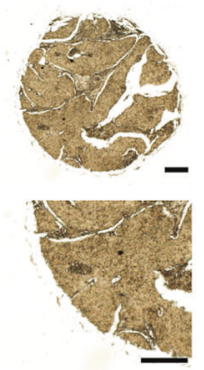

D

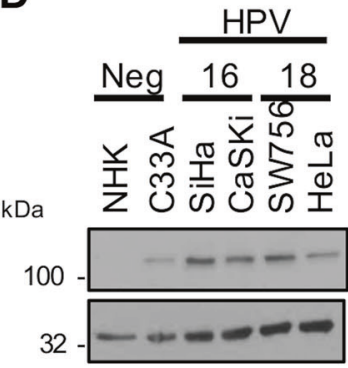

USP13

GAPDH

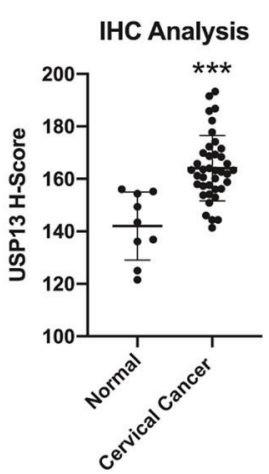

$\mathbf{F}$

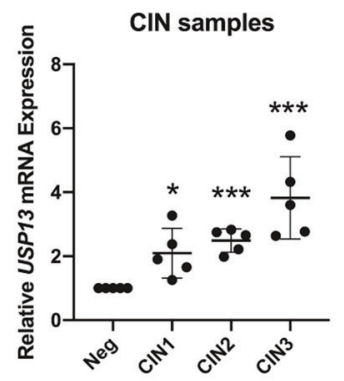

B

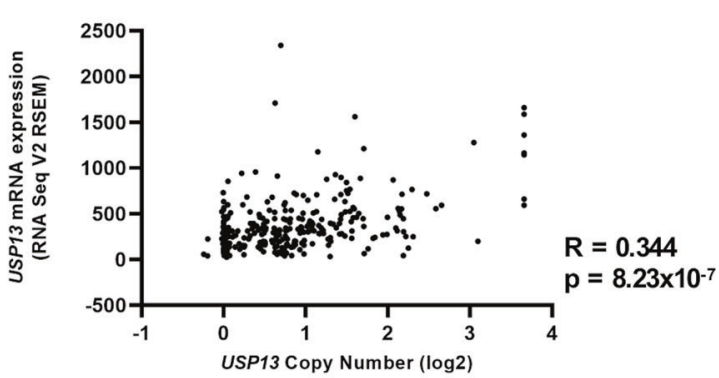

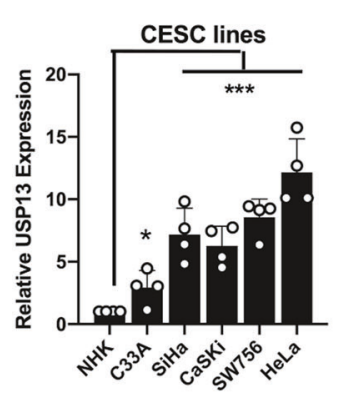

G

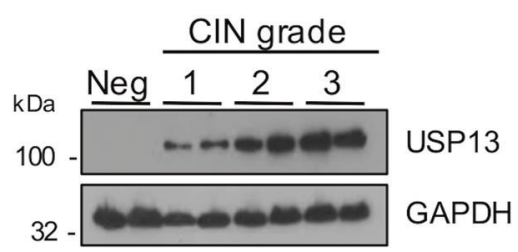

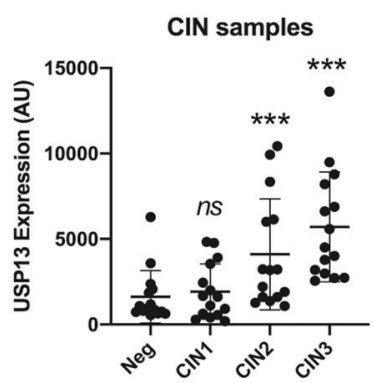

(Fig. 1C, D). To investigate if the HPV oncogenes could directly induce USP13 expression, we first transiently expressed HR-HPV E6 and E7, alone or in combination, in HPV- C33A cells. When expressed alone, neither oncogene increased USP13 expression; co-expression of both E6 and E7 led to a small, but non-significant, increase in USP13 expression. Western blot was used to confirm appropriate expression of each oncoprotein. We did note a lower expression of E6 in the co-expression samples, although it was still detectable using antibodies against E6 and GFP (Supplementary Fig. 3A, B). Furthermore, depletion of endogenous E6/ E7 in HeLa cells did not alter USP13 expression (Supplementary Fig. 3C, D). To confirm our experimental studies, we analysed USP13 expression in the 
Fig. 1 USP13 expression is upregulated in pre-malignant cervical disease and cervical cancer. A Genomic alterations of USP 13 across human cancers determined by cBioportal analysis of TCGA data. B Scatter dot plot analysis of USP13 mRNA expression against USP13 copy number alterations in cervical cancer determined by cBioportal analysis of TCGA data. Correlation was determined using Spearman's analysis. C RT-qPCR analysis of USP13 mRNA expression in normal human keratinocytes (NHKs), HPV- C33A cells, HPV16 + SiHa and CaSKi cells and HPV18 + SW756 and HeLa cells. mRNA expression was normalized against U6 mRNA levels. D Representative western blot of USP13 expression in NHKs, C33A cells, SiHa, CaSKi, SW756 and HeLa cells. GAPDH served as a loading control. Quantification of the protein band intensities from four biological, independent repeats are shown on the right. E Representative immunohistochemical (IHC) staining of USP13 expression in cervical cancer tissues and normal cervical epithelium from a tissue microarray (TMA). Scale bars, $100 \mu \mathrm{m}$. Scatter dot plot analysis of USP13 expression from a larger cohort of cervical cancer cases $(n=41)$ and normal cervical epithelium $(n=9)$ is shown on the right. F Scatter dot plot of RT-qPCR analysis of USP13 mRNA expression from a panel of cervical cytology samples representing CIN lesions of increasing grade. Five samples from each clinical grade (negative (Neg) and CIN I-III) were analysed and mRNA levels were normalized to the negative samples. Samples were normalized against $U 6$ mRNA levels. G Representative western blot of cervical cytology samples of CIN lesions of increasing grade analysed for USP13 protein expression. GAPDH served as a loading control. Scatter dot plot analysis of a larger cohort of samples ( $n=15$ for each grade) is shown on the right. Bars represent the mean \pm standard deviation from at least three biological repeats unless otherwise stated. ${ }^{*} p<0.05 ;{ }^{* *} p<0.01$; ${ }^{* *} p<0.001$ (Student's $t$-test).

TCGA cervical cancer dataset based on HPV status (Supplementary Fig. 3E). In agreement with our cell line data, USP13 expression was not significantly different between HPV + and HPV- cervical cancer. These data together suggest that HPV does not directly modulate USP13 expression in cervical cancer.

\section{USP13 expression is regulated by c-Jun/AP-1 in cervical cancer cells}

In addition to the USP13 amplification observed by our analysis of the TCGA cervical cancer dataset (Fig. 1A), we noted that several cases had high USP13 mRNA expression in the absence of USP 13 amplification. We therefore wished to further investigate how USP 13 expression is regulated in cervical cancer. Whilst oncogenes can be upregulated in cancer at the transcriptional level, the transcriptional regulation of USP13 is poorly understood. To gain insight, we analysed the putative USP13 promoter region upstream of the USP13 start codon for transcription factor binding sites (Fig. 2A). We identified two AP-1 like sequences that have previously been shown to bind to c-Jun, a member of the AP-1 transcription factor family that is highly active in cervical cancer $[37,38]$. To test if c-Jun/AP-1 regulates USP13 expression we first inhibited the activity of the MAP kinase (MAPK) JNK, which is an important inducer of
c-Jun/AP-1 signalling [39]. Addition of the potent, specific irreversible JNK inhibitor JNK-IN-8 abrogated c-Jun phosphorylation and reduced c-Jun expression, as previously observed. Furthermore, we saw a substantial reduction in USP13 protein expression (Fig. 2B). JNK inhibition also decreased USP13 mRNA expression, demonstrating that JNK-mediated c-Jun activity may regulate USP13 at the transcriptional level (Fig. 2C). To investigate if c-Jun directly played a role, we depleted c-Jun expression using a pool of specific siRNAs. Knockdown of c-Jun resulted in a significant decrease in USP13 mRNA and protein expression in both $\mathrm{HeLa}$ and $\mathrm{SiHa}$ cells (Fig. 2D, E). Finally, we assessed if c-Jun regulated USP13 expression directly, by binding the USP 13 promoter via the putative AP-1 binding sites. We performed chromatin immunoprecipitation (ChIP) analysis utilising primers covering the AP-1 like sequences in the USP13 promoter (termed AP-1-1 and AP-1-2; Fig. 2A). In DMSO treated HeLa cells, there was a significant enrichment of c-Jun binding to the AP-1-1 site in the USP13 promoter compared to an IgG isotype control. In contrast, little to no binding of c-Jun was observed at AP-1-2 (Fig. 2F). Crucially, c-Jun binding to the AP-1-1 site was dependent on JNK activity, as treatment with JNK-IN-8 abolished c-Jun binding to the USP13 promoter. Taken together, JNK-dependent c-Jun activation is necessary for USP13 expression in cervical cancer cells.

\section{USP13 is required for HPV + cervical cancer cell proliferation}

Depletion of USP13 using a pool of specific siRNAs (Fig. 3A) caused a significant reduction in HPV + cervical cancer cell growth (Fig. 3B). The absence of USP13 also led to a reduction in colony formation under anchorage dependent (Fig. 3C) and independent conditions (Fig. 3D). In contrast, depletion of USP13 had minimal impact on cell proliferation or colony formation in HPV- cervical cancer cells (Supplementary Fig. 4A-C). Conversely, over-expression of wild type (WT) USP13 enhanced cell growth and colony formation in HPV + cell lines (Fig. 3E-H). This enhancement was dependent on USP13 deubiquitinase activity, as a catalytically inactive mutant of USP13 (USP13 C345A) failed to increase cell growth. Together, these results show that expression of catalytically active USP13 is required for the proliferation of HPV + cervical cancer cells.

\section{USP13 promotes Mcl-1 stability}

Several USP13 targets have been identified, including microphthalmia-associated transcription factor (MITF), ATP citrate lyase (ACLY) and phosphatase and tensin homolog (PTEN) [40-42]. Interestingly, USP13 was 


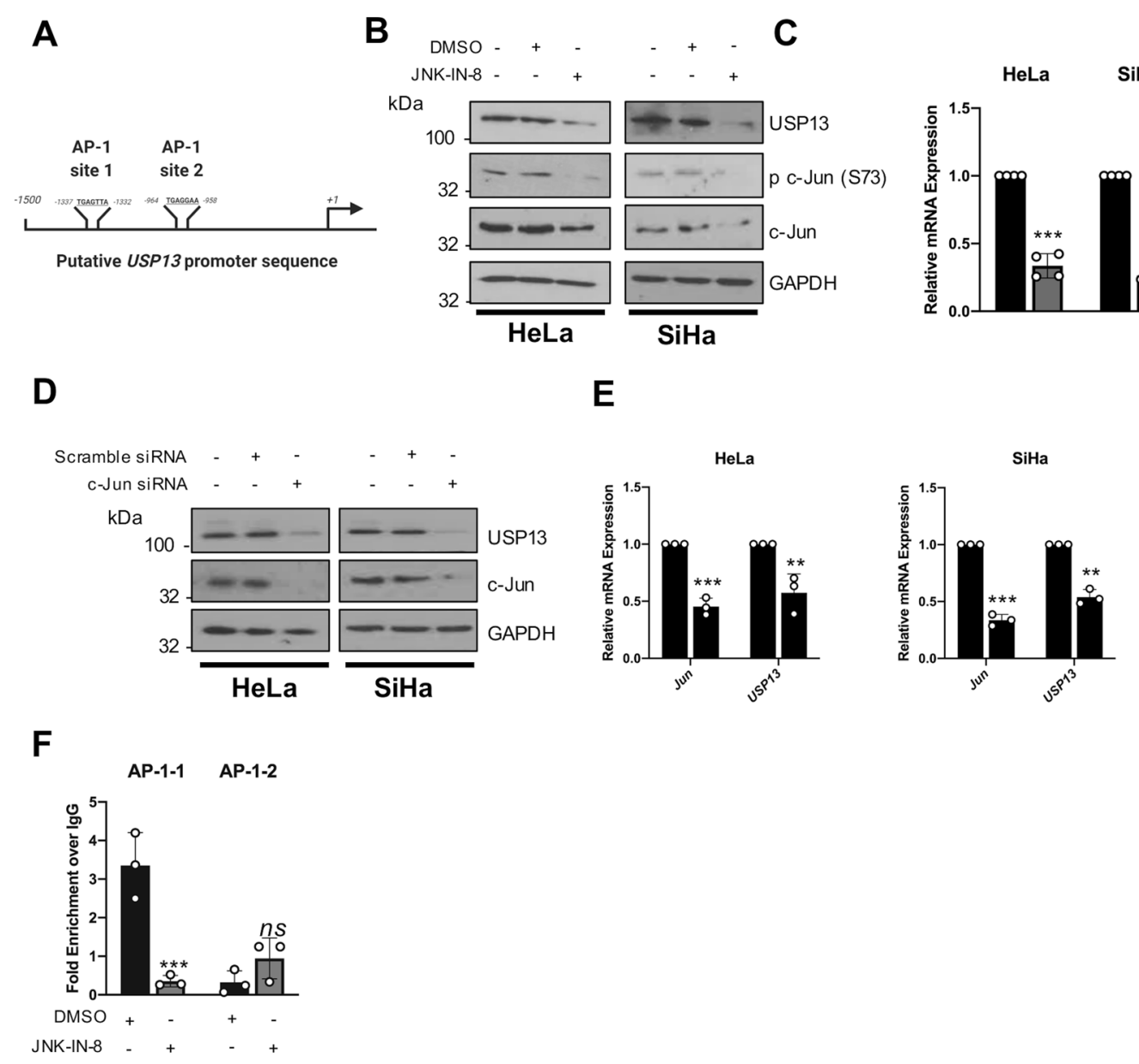

Fig. 2 USP13 expression is regulated by c-Jun/AP-1 activity in cervical cancer cells. A Schematic of potential AP-1 binding sites in the USP13 promoter region. B Representative western blot of HeLa and SiHa cells after treatment with JNK-IN-8 $(3 \mu \mathrm{M})$ or DMSO control for $48 \mathrm{~h}$. Lysates were analysed for the expression of USP13, phosphorylated c-Jun (S73) and c-Jun. GAPDH was used as a loading control. C RT-qPCR analysis of USP13 mRNA expression in HeLa and SiHa cells after treatment with JNK-IN-8 $(3 \mu \mathrm{M})$ or DMSO control for $48 \mathrm{~h}$. mRNA expression was normalized against $U 6 \mathrm{mRNA}$ levels. D Representative western blot of HeLa and $\mathrm{SiHa}$ cells after transfection of a pool of four specific c-Jun siRNA for $72 \mathrm{~h}$. Lysates were

recently shown to deubiquitinate and stabilise the prosurvival protein Mcl-1 in lung and ovarian cancers [43]. Like cervical cancer, ovarian cancers are squamous cell carcinomas; therefore, we hypothesised that USP13 might also regulate Mcl-1 in cervical cancer cells. To investigate this, the level of Mcl-1 expression was analysed in USP13 depleted cells. Loss of USP13 significantly reduced Mcl-1 protein expression, without effecting MCL1 mRNA expression (Fig. 4A, B). Critically, treatment of USP13 depleted cells with the proteasome inhibitor MG132 rescued Mcl-1 protein levels, suggesting that USP13 protects Mcl-1 from proteasomal degradation (Fig. 4C). As a complementary approach, the over-expression of USP13 caused analysed for the expression of USP13 and c-Jun. GAPDH was used as a loading control. E RT-qPCR analysis of USP13 and cJUN mRNA expression of $\mathrm{HeLa}$ and $\mathrm{SiHa}$ after transfection of a pool of four specific c-Jun siRNA for $72 \mathrm{~h}$. mRNA expression was normalized against $U 6$ mRNA levels. $\mathbf{F}$ Cells were treated as in $\mathbf{B}$ and chromatin was prepared from HeLa cells and c-Jun was immunoprecipitated using an anti-c-Jun antibody, followed by RT-qPCR using primers specific to the two putative AP-1 binding sites in the USP 13 promoter. c-Jun binding is presented as percentage of input chromatin. Bars are the means \pm standard deviation from at least three biological repeats. ${ }^{*} p<0.05 ; * *<0.01 ; * * * p<0.001$ (Student's $t$-test).

an increase in Mcl-1 protein levels (Fig. 4D). This was dependant on the catalytic activity of USP13, as a catalytically inactive USP13 mutant did not increase Mcl-1 protein levels (Fig. 4D). Again, USP13 over-expression had no effect on MCL1 mRNA expression (Fig. 4E), suggesting that the regulation of Mcl-1 by USP13 is post transcriptional. Interestingly, over-expression or depletion of USP13 did not modulate Mcl-1 expression in HPV- C33A cells (Supplementary Fig. 5A, B), suggesting that Mcl-1 expression is not regulated by USP13 in these cells. The effect of USP13 on Mcl-1 protein turnover was investigated by cycloheximide chase assay. When USP13 was depleted from HeLa cells, the half-life of Mcl-1 was markedly 
A

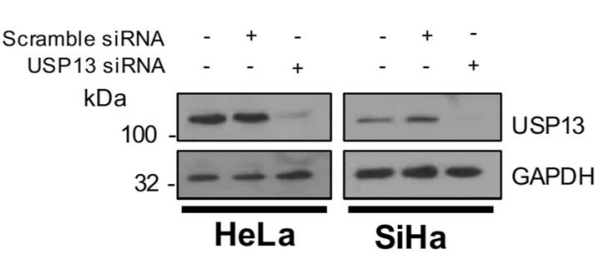

B
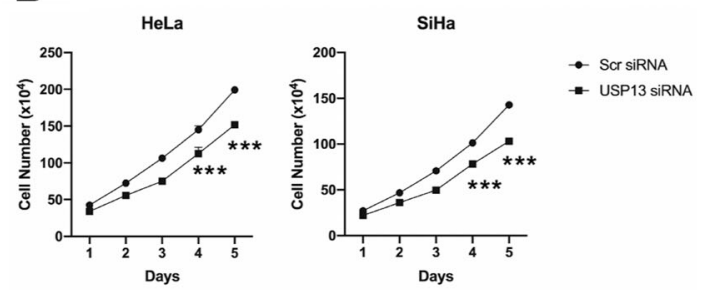
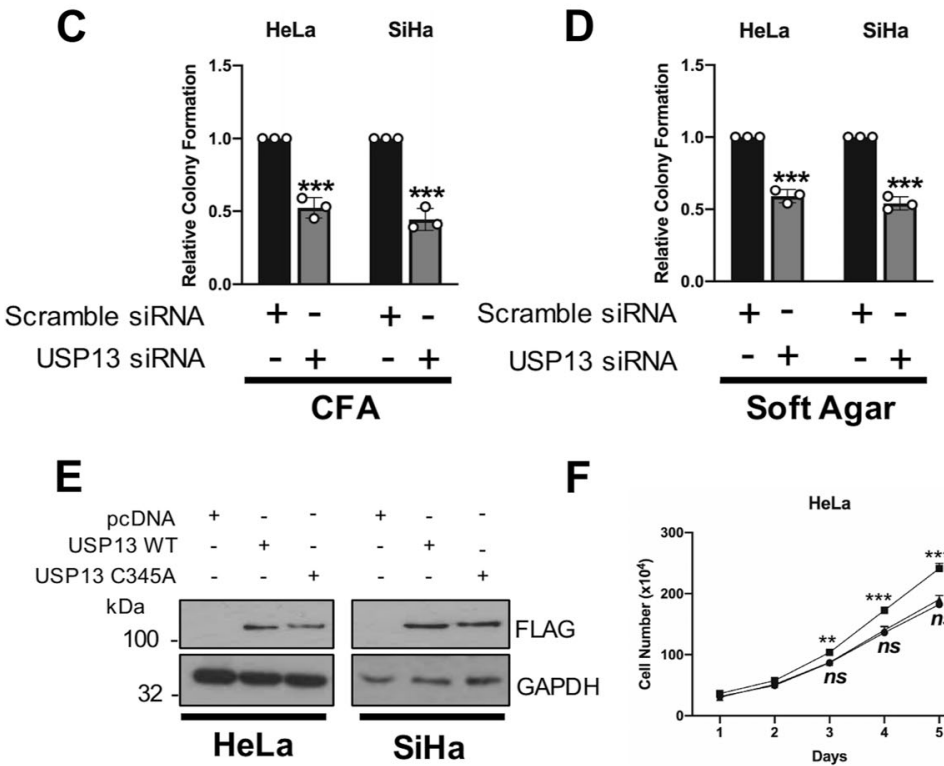

$\mathbf{F}$
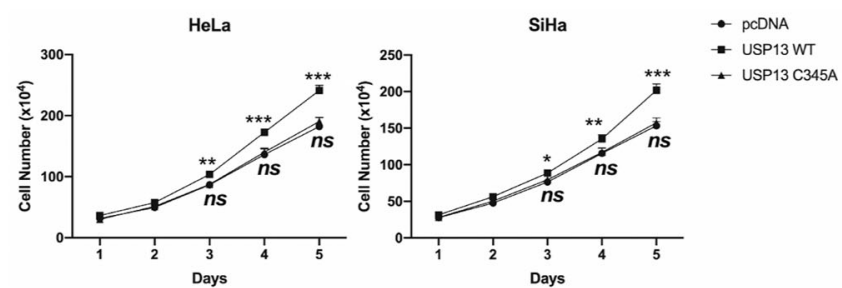

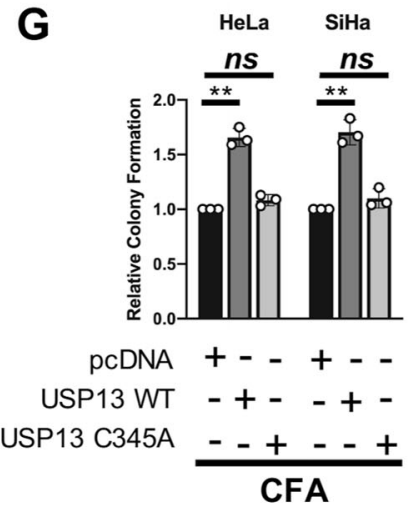

Fig. 3 USP13 expression is required for the proliferation of HPV + cervical cancer cells. A Representative western blot of HeLa and $\mathrm{SiHa}$ cells after transfection of a pool of four specific USP13 siRNA for $72 \mathrm{~h}$. Lysates were analysed for the expression of USP13 and GAPDH was used as a loading control. B Growth curve analysis of $\mathrm{HeLa}$ and $\mathrm{SiHa}$ cells after transfection of a pool of four specific USP13 siRNA for $72 \mathrm{~h}$. C Colony formation assay (anchorage dependent growth) of HeLa and $\mathrm{SiHa}$ cells after transfection of a pool of four specific USP13 siRNA for $72 \mathrm{~h}$. D Soft agar assay of HeLa and SiHa cells after transfection of a pool of four specific USP13 siRNA for $72 \mathrm{~h}$. E Representative western blot of $\mathrm{HeLa}$ and SiHa cells after

reduced, from $\sim 65 \mathrm{~min}$ to $\sim 20 \mathrm{~min}$ (Fig. $4 \mathrm{~F}$ ). Conversely, overexpression of WT USP13, but not the USP13 mutant, enhanced the half-life of Mcl-1 to $\sim 300$ min (Fig. 4G). transfection of a FLAG-USP13 or FLAG-USP13 (C345A) for $48 \mathrm{~h}$ Lysates were analysed for the expression of USP13 and GAPDH was used as a loading control. F Growth curve analysis of HeLa and $\mathrm{SiHa}$ cells after transfection of a FLAG-USP13 or FLAG-USP13 (C345A) for $48 \mathrm{~h}$. G Colony formation assay (anchorage dependent growth) of HeLa and SiHa cells after transfection of a FLAG-USP13 or FLAGUSP13 (C345A) for $48 \mathrm{~h}$. H Soft agar assay of HeLa and SiHa cells after transfection of a FLAG-USP13 or FLAG-USP13 (C345A) for $48 \mathrm{~h}$. Bars are the means \pm standard deviation from at least three biological repeats. $* p<0.05$; $* * p<0.01$; $* * * p<0.001$ (Student's $t$-test).

These data demonstrate that USP13 promotes the stability of Mcl-1 in HPV + cervical cancer cells by protecting it from proteasomal degradation. 


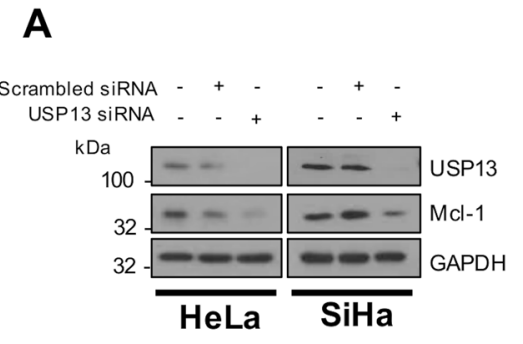

C

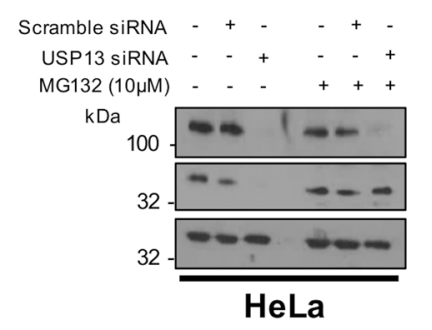

D

$\mathbf{F}$

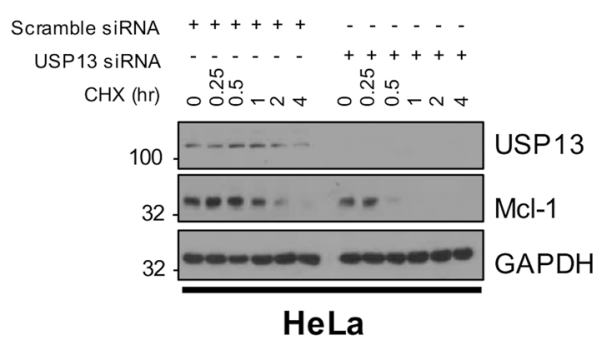

G

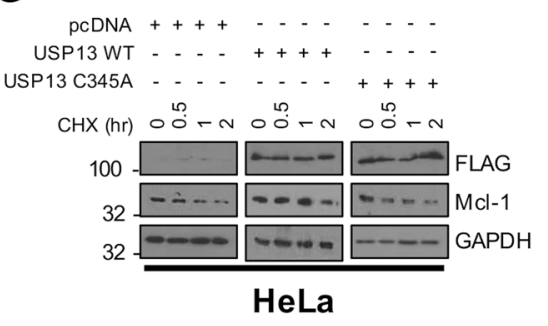

Fig. 4 USP13 promotes the stability of the pro-survival protein Mcl-1. A Representative western blot of HeLa and SiHa cells after transfection of a pool of four specific USP13 siRNA for $72 \mathrm{~h}$. Lysates were analysed for the expression of USP13 and Mcl-1. GAPDH was used as a loading control. B RT-qPCR analysis of USP13 and MCL1 mRNA expression of HeLa and SiHa cells after transfection of a pool of four specific USP13 siRNA for $72 \mathrm{~h}$. mRNA expression was normalized against $U 6 \mathrm{mRNA}$ levels. C Representative western blot of HeLa and SiHa cells after transfection of a pool of four specific USP13 siRNA for $72 \mathrm{~h}$. Cells were additionally treated with $10 \mu \mathrm{M}$ MG132 for $6 \mathrm{~h}$. Lysates were analysed for the expression of USP13 and Mcl-1. GAPDH was used as a loading control. D Representative western blot of $\mathrm{HeLa}$ and $\mathrm{SiHa}$ cells after transfection of FLAGUSP13 or FLAG-USP13 (C345A). Lysates were analysed for the expression of USP13 and Mcl-1. GAPDH was used as a loading control. E RT-qPCR analysis of USP13 and MCL1 mRNA expression of HeLa and SiHa cells after transfection of FLAG-USP13 or FLAG-
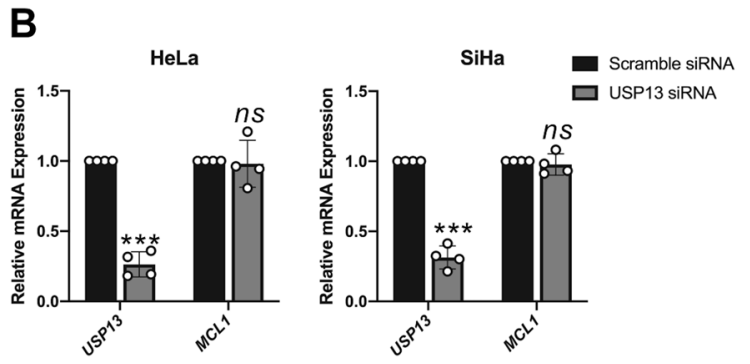

E
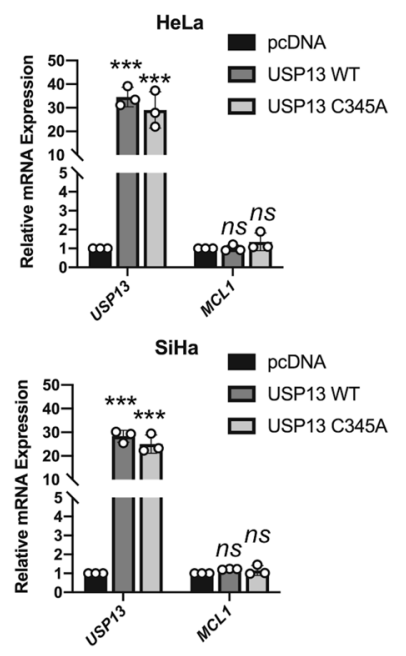


\section{USP13 interacts with and deubiquitinates $\mathrm{Mcl}-1$ in cervical cancer cells}

USP13 has previously been shown to directly bind to Mcl1 in vitro [43]. Furthermore, Mcl-1 has also been shown to be highly expressed in cervical cancer and correlates with proliferation and cell survival [44]. We first performed coimmunoprecipitation assays to confirm the interaction between USP13 and Mcl-1 by transfecting HEK293T cells with FLAG-USP13 and V5-hMcl-1. Immunoprecipitation with anti-FLAG antibody demonstrated that FLAGUSP13 interacted with over-expressed Mcl-1 (Fig. 5A). Furthermore, reciprocal immunoprecipitations using antiV5 antibody confirmed this interaction (Fig. 5A). To identify if this interaction also occurred between endogenous USP13 and Mcl-1 in cervical cancer cells, immunoprecipitations were performed with either antiUSP13 or anti-Mcl-1 antibodies in HeLa and SiHa cells lysates. Immunoprecipitation with either antibody resulted in the detection of both USP13 and Mcl-1, suggesting that the endogenous proteins interact in cervical cancer cells (Fig. 5B). Next, we confirmed that USP13 deubiquitinated Mcl-1. HEK293T cells were transfected with FLAGUSP13, V5-Mcl-1 and HA-Ubiquitin, and anti-V5 immunoprecipitates were probed for the level of ubiquitination using the HA-antibody. Co-expression of USP13 and Mcl-1 led to a marked reduction in Mcl-1 ubiquitination (Fig. 5C, compare lanes 2 and 3). Consistent with our previous data, the catalytically inactive USP13 mutant was not able to reduce Mcl-1 ubiquitination, despite retaining the ability to bind to Mcl-1 (Fig. 5C, compare lanes 2 and 4; Supplementary Fig. 6). Whilst in vitro ubiquitination assays confirmed that USP13 is capable of directly deubiquitinating Mcl-1 [43], the precise ubiquitin linkage that USP13 removes from Mcl-1 has not been determined. Ubiquitin contains seven lysine residues that serve as sites of ubiquitination. The most studied types are K48 polyubiquitin, which targets proteins for degradation, and K63 polyubiquitin, which is predominantly associated with signal transduction [3]. To determine which ubiquitin linkage USP13 deubiquitinates, HEK293T cells were transfected with the HA-tagged K48R or K63R ubiquitin mutants, which are unable to be polyubiquitinated with K48 or K63 linkages, respectively. Overexpressed USP13 was able to cleave wild-type and the K63R form of ubiquitin from Mcl-1 but not K48R ubiquitin (Fig. 5D). These results suggest that USP13 removes K48 polyubiquitin, but not K63 polyubiquitin, chains from Mcl-1. Finally, we confirmed that depletion of USP13 from both $\mathrm{HeLa}$ and $\mathrm{SiHa}$ cells increased the ubiquitination of endogenous Mcl-1, suggesting USP13-mediated deubiquitination of Mcl-1 is relevant in cervical cancer cells (Fig. 5E).

\section{Restoration of $\mathrm{Mcl}-1$ expression partially rescues the proliferation defect in USP13 depleted cervical cancer cells}

As well as having a critical role in cell survival, Mcl-1 can also play an important role in cell proliferation [45]. As USP13 depletion resulted in the inhibition of cell proliferation in cervical cancer cells, we investigated if this was due to the reduction in Mcl-1 levels. We therefore over-expressed Mcl-1 alone or in in USP13-depleted cells (Fig. 6A). Expression of Mcl-1 alone led to a modest increase in cell proliferation and colony formation (Fig. 6B). Importantly, the re-introduction of Mcl-1 in USP13-depleted cells resulted in a partial restoration of proliferation (Fig. 6B) and colony formation (Fig. 6C). These results suggest that the effect of USP13 on cell proliferation in cervical cancer cells is at least partially dependent on the stabilisation of Mcl-1 expression.

\section{USP13 and Mcl-1 expression correlate in cervical cancer}

To confirm the clinical relevance of the USP13 - Mcl-1 interaction, we first analysed the expression of Mcl-1 in our panel of cervical cancer cells. Mcl-1 protein levels were significantly higher in cervical cancer cells when compared to NHKs (Fig. 7A) and correlated with increased USP13 expression in the cervical cancer cell lines $(R=0.862$, $p=0.015)$. We assessed Mcl-1 expression in cervical cancer tissue by immunohistochemistry and observed higher expression of Mcl-1 in cervical cancer tissue when compared to non-cancer tissue (Fig. 7B, C). Importantly, expression of Mcl-1 significantly correlated with USP13 expression in cervical cancer tissue from the same patient (Fig. 7D; $R=0.4128, p=0.009$ ). Collectively, these findings demonstrate that USP13 and Mcl-1 are concomitantly over expressed in cervical cancer tissue, suggesting that the regulation of Mcl-1 by USP13 is clinically relevant.

\section{Inhibition of USP13 sensitises cervical cancer cells to BH3 mimetics}

BCL-2 family members are highly expressed in cervical cancer and inhibition of these proteins using $\mathrm{BH} 3$ mimetics is a promising therapeutic strategy [46]. Despite this promise, high expression levels of Mcl-1 is commonly associated with resistance to these inhibitors and inhibition or depletion of Mcl-1 can restore sensitivity to $\mathrm{BH} 3$ mimetics [47]. To investigate if targeting USP13 to reduce Mcl-1 expression has therapeutic potential in cervical cancer, we first assessed the impact of USP13 inhibition by the small molecule inhibitor Spautin-1 [48]. In both HeLa and SiHa cells, inhibition of USP13 by Spautin-1 resulted in a dosedependent decrease in Mcl-1 protein expression (Fig. 8A). 


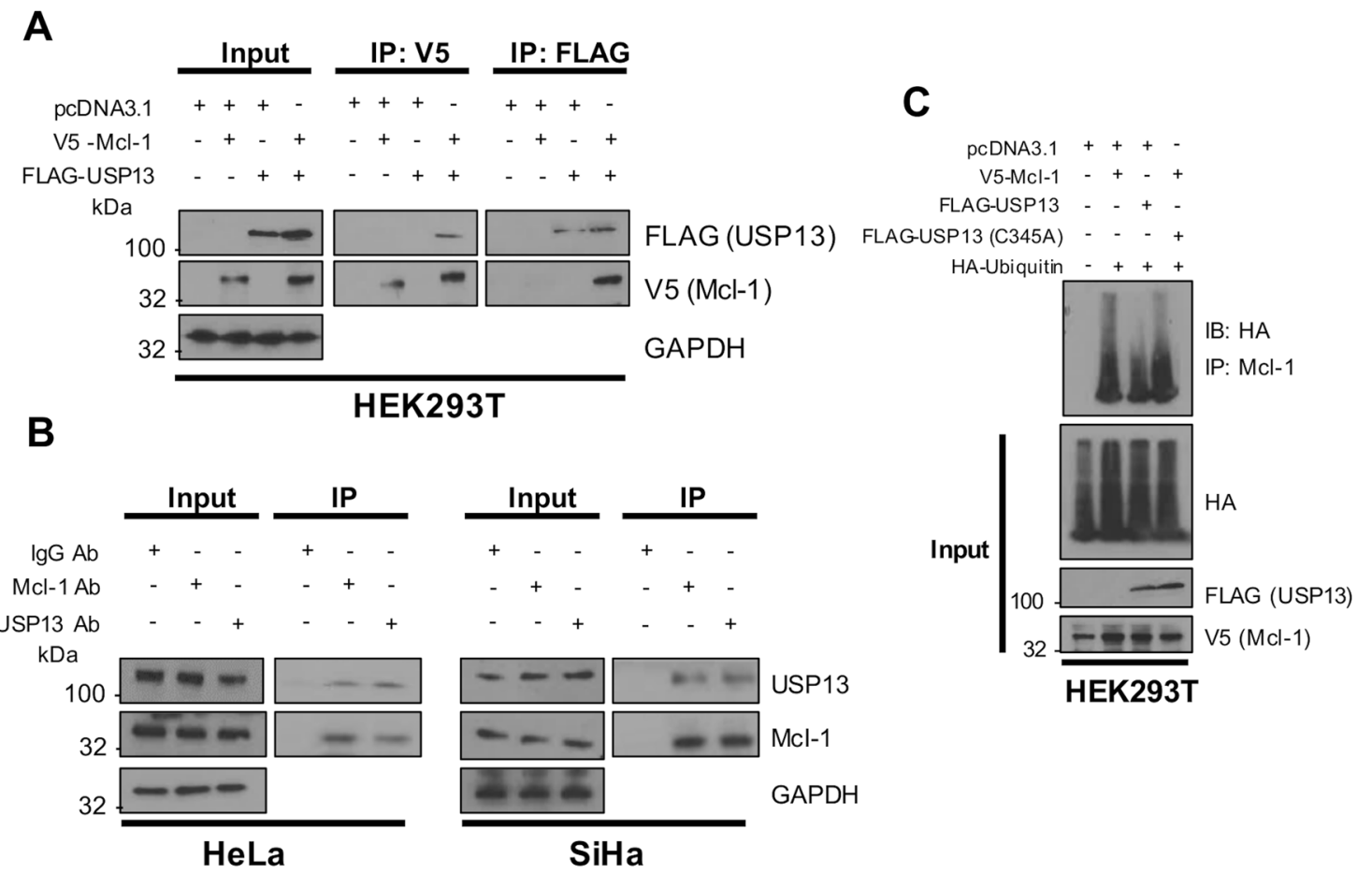

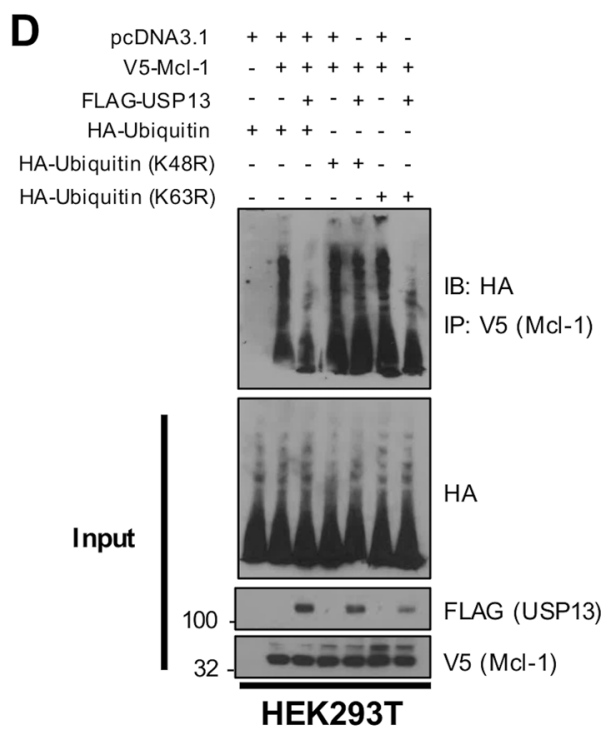

Fig. 5 USP13 interacts with and deubiquitinates Mcl-1 in cervical cancer cells. A HEK293T cells were transfected with V5-Mcl-1, FlagUSP13, or both V5-Mcl-1 and FLAG-USP13. Cells were treated with $10 \mu \mathrm{M}$ MG132 for $6 \mathrm{~h}$ and either Mcl-1 or USP13 were immunoprecipitated using an anti-V5 or anti-FLAG antibody. Coimmunoprecipitated Mcl-1 or FLAG-USP13 were detected using the respective antibodies. GAPDH was used as a loading control. B Endogenous Mcl-1 and USP13 were immunoprecipitated from $\mathrm{HeLa}$ and SiHa cells after treatment with $10 \mu \mathrm{M}$ MG132 using an antiMcl-1 or anti-USP13 antibodies. Co-immunoprecipitated Mcl-1 or USP13 were detected using the respective antibodies. GAPDH was used as a loading control. C HEK293T cells were co-transfected with V5-Mcl-1 and HA-Ubiquitin, with or without Flag-USP13 or a FLAG-
E

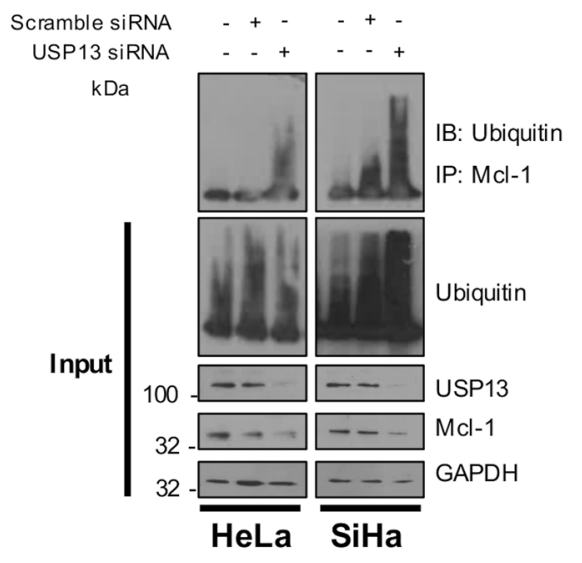

USP13 mutant (C345A). Cells were treated with $10 \mu \mathrm{M} \mathrm{MG132}$ for $6 \mathrm{~h}$ and V5-Mcl-1 was immunoprecipitated using an anti-Mcl-1 antibody. Ubiquitinated Mcl-1 was detected using an anti-HA antibody. D HEK293T cells were co-transfected with V5-Mcl-1, HA-Ubiquitin or mutant Ubiquitin (K48R or K63R), with or without Flag-USP13. Cells were treated with $10 \mu \mathrm{M}$ MG132 for $6 \mathrm{~h}$ and V5-Mcl-1 was immunoprecipitated using an anti-V5 antibody. Ubiquitinated Mcl-1 was detected using an anti-HA antibody. E HeLa and SiHa cells were transfected with a pool of four specific USP13 siRNA for $72 \mathrm{~h}$. Cells were treated with $10 \mu \mathrm{M}$ MG132 for $6 \mathrm{~h}$ before harvesting. Mcl-1 was immunoprecipitated using an anti-Mcl-1 antibody. Ubiquitinated Mcl1 was detected using an anti-ubiquitin antibody. GAPDH was used as a loading control. 


\section{A}

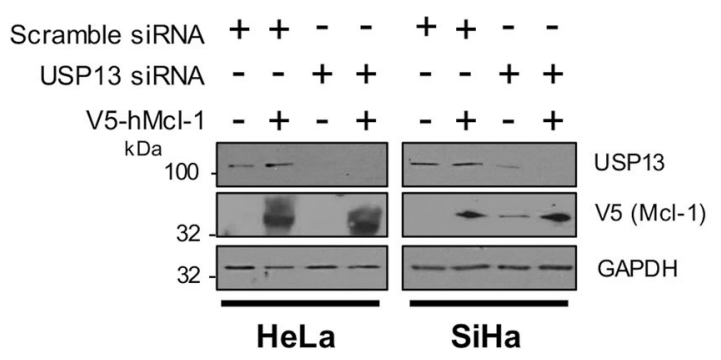

C

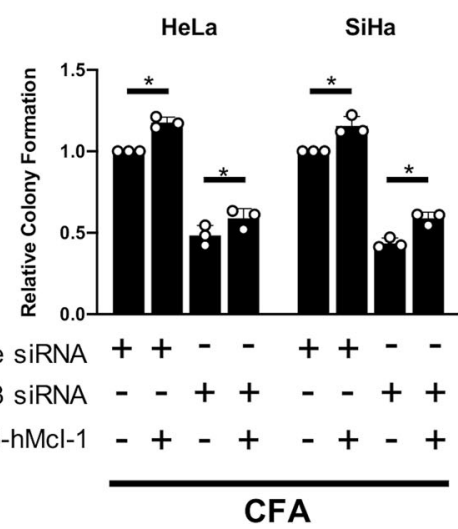

Fig. 6 Restoration of Mcl-1 expression partially rescues the proliferation defect in USP13 depleted cervical cancer cells. A Representative western blot of HeLa and $\mathrm{SiHa}$ cells after transfection of a pool of four specific USP13 siRNA, or scramble siRNA, for $72 \mathrm{~h}$. After $24 \mathrm{~h}$, cells were transfected with hMcl-1 or control vector. Lysates were analysed for the expression of USP13, Mcl-1 and GAPDH was used as a loading control. B Growth curve analysis of $\mathrm{HeLa}$ and $\mathrm{SiHa}$ cells after transfection of a pool of four specific

Spautin-1 also reduced USP13 expression, as has been previously observed in other cell lines (Fig. 8A). Similar to USP13 depletion, treatment with Spautin-1 had no effect on MCL1 mRNA expression (Fig. 8B). Furthermore, the reduction in Mcl-1 protein expression upon Spautin-1 treatment was lessened by inhibition of the proteasome (Fig. 8C). Treatment with Spautin-1 also abolished the increase in Mcl-1 expression observed upon USP13 overexpression, suggesting that the function of Spautin-1 may be through the inhibition of USP13 (Fig. 8D). Cervical cancer cells are inherently resistant to the $\mathrm{BH} 3$ mimetic ABT-263 [49]. We therefore investigated if pharmacological inhibition of USP13 using Spautin-1 sensitised cervical cancer cells to ABT-263. In line with previous reports, $\mathrm{HeLa}$ and $\mathrm{SiHa}$ cells were relatively insensitive to ABT263 , with an $\mathrm{IC}_{50}$ of 10.64 and $18.58 \mu \mathrm{M}$, respectively (Fig. 8E). However, combination treatment with Spautin-1 significantly sensitised cervical cancer cells to ABT-263 (HeLa, 12-fold increase, $10.64 \mu \mathrm{M}$ to $0.89 \mu \mathrm{M}$; SiHa, 15fold increase, $18.58 \mu \mathrm{M}$ to $1.32 \mu \mathrm{M}$ ). Furthermore, in line with our proliferation data, Spautin-1 did not significantly alter the response of HPV- C33A cells to ABT-263
B
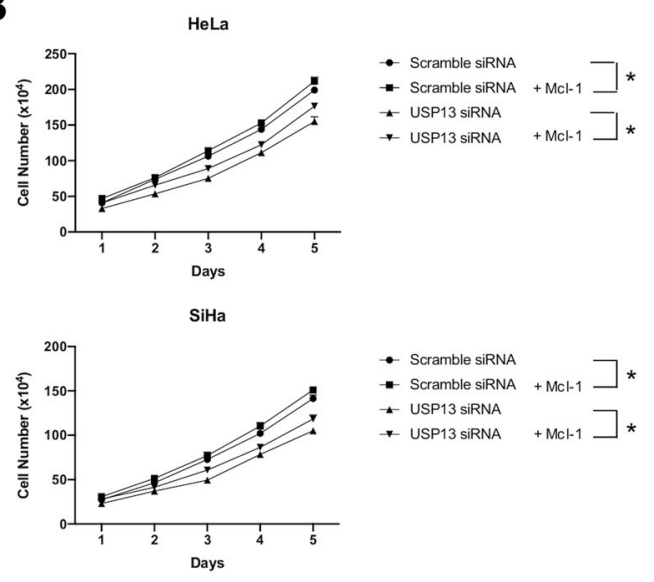

USP13 siRNA, or scramble siRNA, for $72 \mathrm{~h}$. After $24 \mathrm{~h}$, cells were transfected with hMcl-1 or control vector. C Colony formation assay (anchorage dependent growth) of HeLa and SiHa cells after transfection of a pool of four specific USP13 siRNA, or scramble siRNA, for $72 \mathrm{~h}$. After $24 \mathrm{~h}$, cells were transfected with hMcl-1 or control vector. Bars are the means \pm standard deviation from at least three biological repeats. ${ }^{*} p<0.05 ; * * p<0.01 ; * * * p<0.001$ (Student's $t$-test).

treatment (Supplemantary Fig. 7; ABT-263 IC $_{50} 11.56 \mu \mathrm{M}$ vs $10.25 \mu \mathrm{M}$ with Spautin-1 co-treatment). To expand on this, we performed dose matrix experiments containing a range of ABT-263 and Spautin-1 concentrations and assessed potential drug synergy using the Bliss independence model [50]. An overall negative Bliss synergy score indicates antagonism; a value of zero indicates additive activity, and positive Bliss synergy score indicates synergy. The heatmaps for Bliss synergy scores showed that ABT263 in combination with Spautin-1 synergistically inhibited cell viability across multiple concentrations (Fig. 8F).

As a single agent, ABT-263 did not induce apoptosis in cervical cancer cells, whereas Spautin-1 alone did, particularly in SiHa cells (Fig. 8G). Combination treatment resulted in around a 15-fold increase in apoptosis in both cell lines (Fig. 8G). Finally, we demonstrated that whilst Spautin-1 reduced colony formation by around 50\% (similar to USP13 depletion), combination treatment with ABT-263 resulted in a $75 \%$ reduction in colony formation (Fig. $8 \mathrm{H}$ ). Taken together, these data suggest that USP13 mediated stabilisation of Mcl-1 may be a potential mechanism for the resistance of cervical cancer cells to $\mathrm{BH} 3$ mimetics. 
A

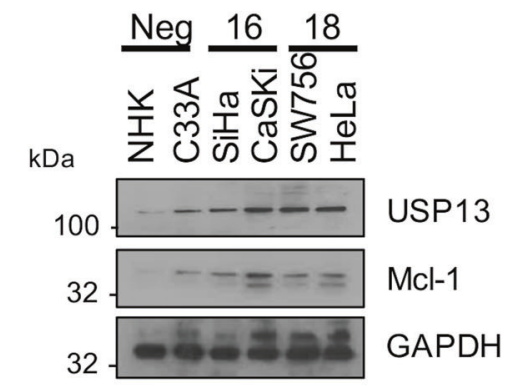

D

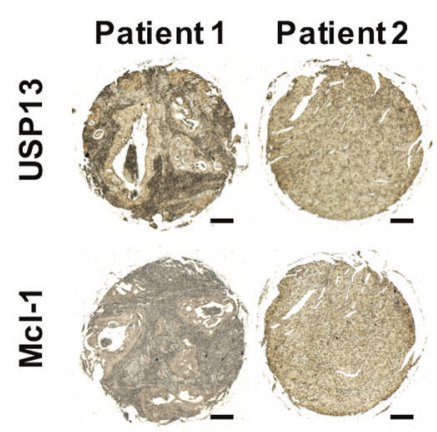

B

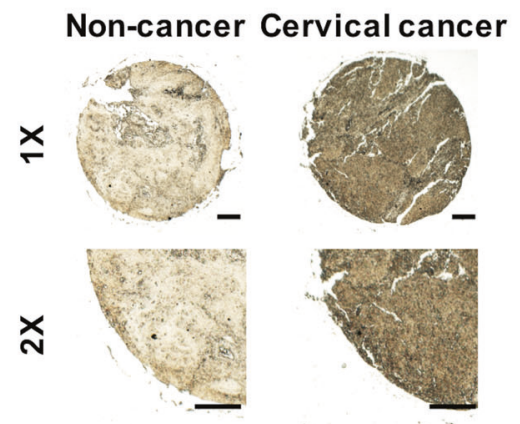

C

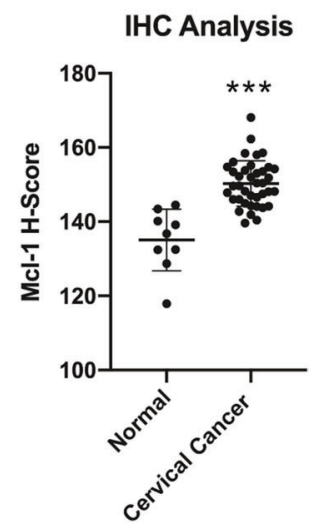

Fig. 7 USP13 and Mcl-1 expression correlate in cervical cancer. A Representative western blot of Mcl-1 expression in normal human keratinocytes (NHKs), C33A, SiHa, CaSKi, SW756 and HeLa cells. GAPDH served as a loading control. B Representative IHC staining of Mcl-1 expression in cervical cancer tissues and normal cervical epithelium from a tissue microarray (TMA). Scale bars, $100 \mu \mathrm{m}$. C Scatter dot plot analysis of Mcl-1 expression from a larger cohort of cervical

\section{Discussion}

Deregulation of the ubiquitin system is a common occurrence in diverse cancers [51]. As such, targeting the enzymes responsible for either the addition or removal of ubiquitin offers a potential therapeutic avenue. Encouragingly, a number of small molecule inhibitors of ubiquitinassociated enzymes are currently entering pre-clinical trials [52-56]. Here, we screened the TCGA cervical cancer database for the expression of DUBs, enzymes which catalyse the remove of ubiquitin from protein substrates [5]. We identified that USP13 is the most amplified DUB gene in cervical cancer. Interestingly, USP13 sits on chromosome $3 q 26.33$, in the so-called 3q26 'OncCassette', a common amplicon that is found in most squamous cell carcinomas and includes the oncogene PIK3CA [57]. In addition, the TCGA cervical cancer database shows minimal mutations in the USP13 gene (data not shown).

Several substrates for USP13 have recently been identified; however, due to this, the function of USP13 in tumourigenesis is controversial, as it has been shown to
IHC Analysis

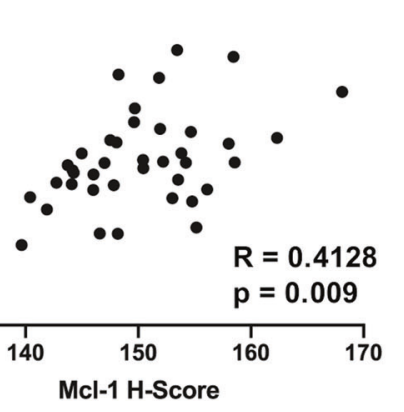

cancer cases $(n=41)$ and normal cervical epithelium $(n=9)$ is shown on the right. D Representative IHC staining of USP13 and Mcl-1 expression in cervical cancer tissue from two patients. Staining was performed from separate cores from the same patient samples. Scale bars, $100 \mu \mathrm{m}$. Correlation was determined using Spearman's analysis and is shown on the right. ${ }^{*} p<0.05$; ${ }^{* *} p<0.01$; $* * * p<0.001$ (Student's $t$-test).

regulate the expression of both tumour suppressors and oncogenes. USP13 can induce Beclin-1 mediated autophagy as part of a p53 regulatory loop [48] and stabilise the tumour suppressor PTEN in breast cancer [41]. In contrast, USP13 also deubiquitinates and stabilises microphthalmiaassociated transcription factor (MITF), an important lineage-specific master regulator in melanoma [40, 58]. Furthermore, USP13 can deubiquitinate and stabilise ACLY/OGDH and c-Myc in ovarian cancer and glioblastoma, respectively, thereby functioning as an oncogene $[42,59]$. Indeed, USP13 depletion did induce a proliferation defect in some ovarian cancer cells but not others, and this was dependent on USP13 expression levels [42, 43]. Interestingly, despite the fact that the viral oncogenes do not regulate USP13 expression, depletion of USP13 in HPVC33A cells did not cause a proliferation defect (Supplementary Fig. 4). This may be due to the fact that USP13 did not seem to regulate Mcl-1 in these cells (Supplementary Fig. 5). The reason for the this is unclear at this point; further studies are required in a larger panel of HPV- cervical cancer cells to ascertain a mechanism for the effects 

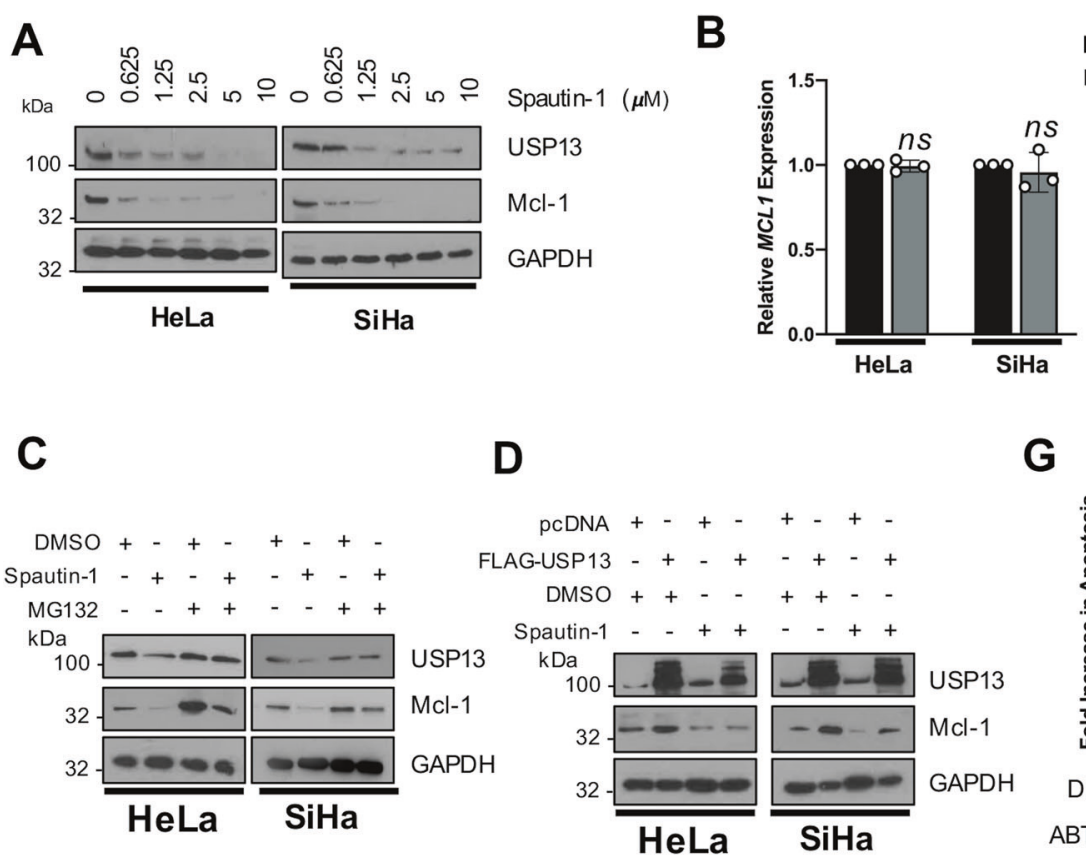
DMSO

E

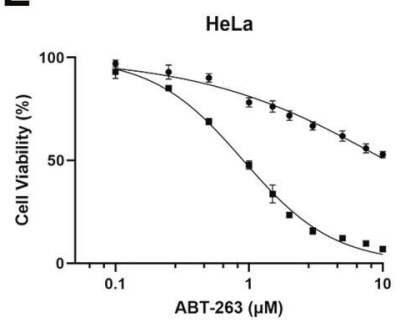

$\mathbf{F}$
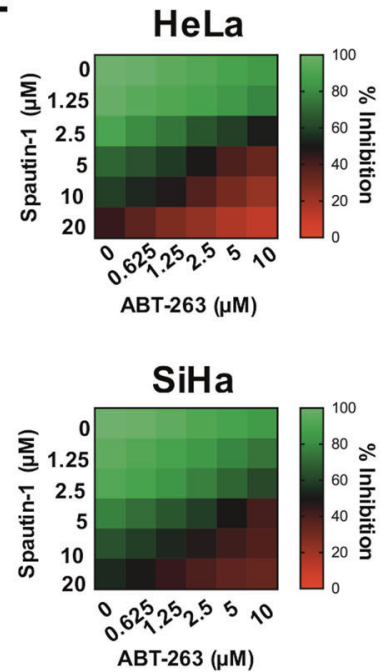
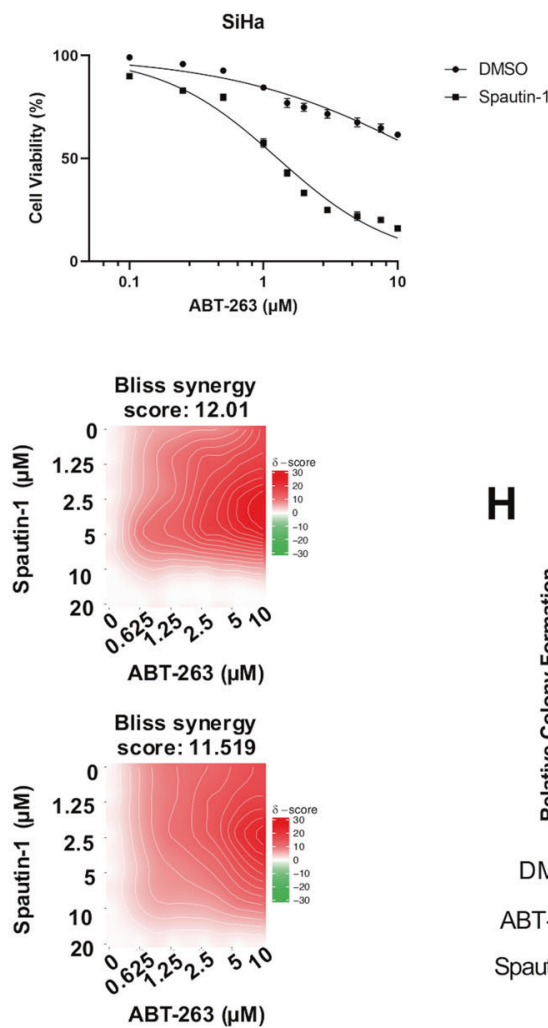

H
G
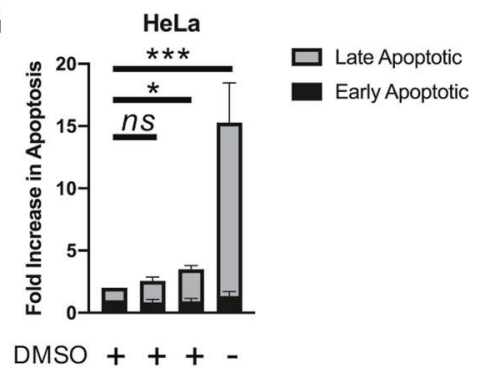

ABT-263 - + - +

Spautin-1 - ++

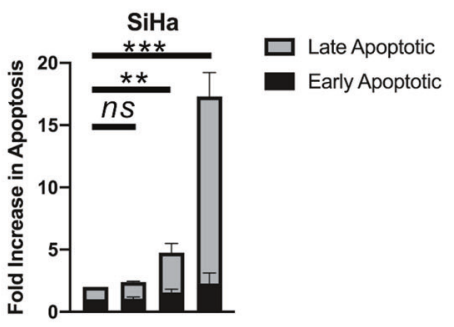

$\mathrm{DMSO}+\boldsymbol{+}+$

ABT-263 - + - +

Spautin-1 - -++

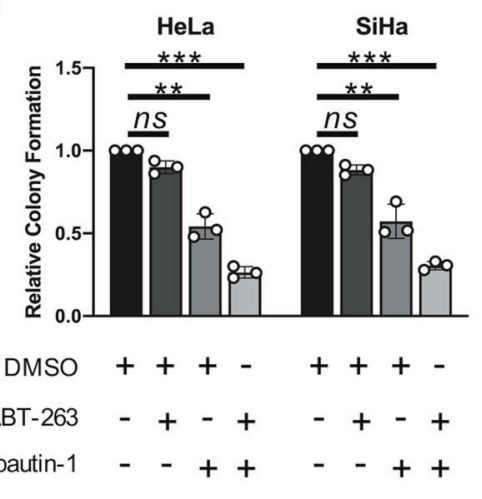

observed in this study. As well as being regulated via copy number alterations, genes can also be regulated at a transcriptional level. The cervical cancer TCGA dataset demonstrates that $8 \%$ of cervical cancers have high USP 13 mRNA expression in the absence of USP13 copy number amplification. We therefore investigated how USP13 expression was regulated in cervical cancer cells. We identified that the USP13 promoter contains two AP-1-like sequences that have previously been shown to bind to the AP-1 family member c-Jun [37, 38]. In this study, we demonstrated that inhibition of JNK, or depletion of c-Jun, resulted in a substantial loss of USP13 expression and this was regulated at the transcriptional level (Fig. 2B-E). Furthermore, chromatin immunoprecipitation assays 
Fig. 8 Pharmacological inhibition of USP13 sensitises HPV + cervical cancer cells to BH3 mimetics. A Representative western blot of $\mathrm{HeLa}$ and $\mathrm{SiHa}$ cells after treatment with increasing doses of Spautin-1 or DMSO control for $48 \mathrm{~h}$. Lysates were analysed for the expression of USP13 and Mcl-1. GAPDH was used as a loading control. B RT-qPCR analysis of MCL1 mRNA expression in HeLa and SiHa cells after treatment with Spautin-1 $(10 \mu \mathrm{M})$ or DMSO control for $48 \mathrm{~h}$. mRNA expression was normalized against $U 6 \mathrm{mRNA}$ levels. C HeLa and SiHa cells were treated with Spautin-1 $(10 \mu \mathrm{M})$ or DMSO control for $48 \mathrm{~h}$. Cells were additionally treated with $10 \mu \mathrm{M}$ MG132 for $6 \mathrm{~h}$. Lysates were analysed for the expression of USP13 and Mcl-1. GAPDH was used as a loading control. D Representative western blot of HeLa and SiHa cells after transfection of FLAG-USP13 with or without Spautin-1 $(10 \mu \mathrm{M})$ or DMSO control. Lysates were analysed for the expression of USP13 and Mcl-1. GAPDH was used as a loading control. E Cell viability assay (MTT) of HeLa and SiHa cells treated with increasing doses of ABT-263, with or without Spautin-1 $(10 \mu \mathrm{M})$, for $24 \mathrm{~h}$. F Synergistic activities of Spautin-1 and ABT-263 in HeLa and SiHa cells. Left: heatmaps of growth inhibition; right: Bliss synergy map. G Flow cytometric analysis of Annexin V assay in $\mathrm{HeLa}$ and SiHa cells after treatment with DMSO, Spautin-1 $(10 \mu \mathrm{M})$, ABT-263 $(5 \mu \mathrm{M})$ or both for $24 \mathrm{~h}$. H Colony formation assay of HeLa and SiHa cells after treatment with DMSO, Spautin-1 $(10 \mu \mathrm{M})$, ABT$263(5 \mu \mathrm{M})$ or both for $24 \mathrm{~h}$. Error bars represent the mean \pm standard

demonstrated that c-Jun directly bound one of the identified AP-1-like sequences in the USP13 promoter (Fig. 2A, F). Thus, we have identified an additional mechanism of USP13 regulation. The BCL-2 family is composed of proapoptotic and anti-apoptotic proteins that play a critical role in regulating cell survival. Mcl-1, along with other proteins such as BCL-2 and BCL-XL, are anti-apoptotic members of the BCL-2 family and have been shown to promote cell survival, chemotherapeutic resistance and are frequently dysregulated in a variety of human cancers (reviewed in [60]). Thus, the regulation of these proteins may be a potential therapeutic target in several cancers. Here, we provide evidence that USP13 functions as an oncogene in cervical cancer by promoting cell proliferation and further demonstrate that this is linked with the stabilisation of the pro-survival BCL-2 family member Mcl-1, by specifically removing K48 polyubiquitin chains that are associated with proteasomal degradation.

In lung and ovarian cancers, where USP13 also regulates Mcl-1 stability, USP13 does not contribute to the cell proliferation of unstressed cells (Fig. 3). Interestingly, restoration of Mcl-1 expression only partially restored cell proliferation in USP13 depleted cervical cancer cells. This suggests that additional USP13 substrates may contribute to cervical cancer cell proliferation. Further studies will be required to identify these targets and understand how they contribute to USP13-mediated cell proliferation. Over recent years, Mcl-1 has attracted attention as a potential therapeutic target in cancer [61, 62]. Mcl-1 plays a critical role as a pro-survival factor, as well as a pro-proliferative factor, in many tumour types. Furthermore, Mcl-1 turnover can be controlled by a number of E3 ligases (such as
$\mathrm{SCF}^{\beta-\mathrm{TrCP}}, \mathrm{SCF}^{\mathrm{FBW} 7}$ and Mule) and DUBs (such as USP9X and DUB3) (reviewed in [63]). Previously, the DUBs OTUD1 and JOSD1 had been shown to regulate Mcl-1 expression in cervical cancer cells $[64,65]$. In contrast to the data presented here, one of these studies did not find a role for USP13 in the regulation of Mcl-1 in HeLa cells [64]; however, that study focussed on the overexpression of USP13. In contrast, we have taken a more holistic approach and assessed the role of USP13 in Mcl-1 stabilisation with a range of complementary assays, providing robust evidence that USP13 can indeed regulate Mcl-1 expression in cervical cancer cells. Furthermore, whilst our data demonstrates that USP13 plays a role in regulating cervical cancer cell proliferation via the stabilisation of Mcl-1, it is clear that several other DUBs play complimentary roles in regulating Mcl-1 expression that may also play pro-oncogenic roles in cervical cancer. As BCL-2 family members have been reported to be highly expressed in cervical cancer, BH3 mimetics may have therapeutic potential in these cancers and the high expression of Mcl-1 has been implicated as a resistance mechanism to these inhibitors [46, 47]. Recent advances have resulted in the development of ABT737 , a potent $\mathrm{BH} 3$ mimetic which disrupts the interaction between anti-apoptotic and pro-apoptotic BCL-2 proteins [66]. In addition, the orally bioavailable analogue ABT-263 (navitoclax) was evaluated in clinical trials and resulted in significant anti-tumour activity [67]. Subsequently, ABT199 (venetoclax) was developed, a highly selective BCL-2 inhibitor that has been approved for patients with chronic lymphocytic leukemia (CLL) harbouring $17 \mathrm{p}$ deletion who have received at least one prior treatment [68]. Despite their promise, an inherent problem with the current $\mathrm{BH} 3$ mimetics, is the development of intrinsic resistance due to high expression of Mcl-1, which these inhibitors do not target $[69,70]$. Therefore, reducing Mcl-1 expression, by direct or indirect inhibition, can sensitise resistant cancer cells to BH3 mimetics [70, 71]. As such, Mcl-1 specific inhibitors, such as AMG 176, can synergise with BH3 mimetics to achieve tumour regression in acute myeloid leukemia (AML) tumour models [69]. Furthermore, studies with cyclin-dependent kinase (CDKs) inhibitors such as alvocidib, or multi-kinase inhibitors such as Sorafenib have shown similar results [72, 73].

Our data shows that USP13 and Mcl-1 protein expression correlates, suggesting that USP13-mediated Mcl-1 stabilisation is clinically relevant. Furthermore, we demonstrated that this mechanism could be exploited pharmacologically, by use of a recently identified small molecule inhibitor of USP13 to reduce Mcl-1 protein expression [48]. This may have potential benefit in cervical cancer patients, as although standard chemotherapeutic options in cervical cancer have good efficacy, many cases develop local recurrences and metastases [74]. Our data demonstrate that inhibition of 
USP13 with Spautin-1 reduces Mcl-1 expression and sensitises cervical cancer cells to the BH3 mimetic ABT-263, offering a potential therapeutic strategy for cervical cancer. In conclusion, we have identified that the DUB USP13 is a potential oncogene in cervical cancer that promotes proliferation by deubiquitinating and stabilising the pro-survival protein Mcl-1. We also demonstrate that pharmacological inhibition of USP13 sensitises cervical cancer cells to a $\mathrm{BH} 3$ mimetic inhibitor by reducing $\mathrm{Mcl}-1$ protein expression, inducing cell death. Therefore, we suggest that targeting USP13 may offer a therapeutic benefit in cervical cancer patients via the reduction in $\mathrm{Mcl}-1$ protein expression.

\section{Materials and methods}

\section{Cervical cytology samples}

Cervical cytology samples were obtained from the Scottish HPV Archive (http://www.shine/mvm.ed.ac.uk/archive.shtml), a biobank of over 20,000 samples designed to facilitate HPV research. The East of Scotland Research Ethics Service has given generic approval to the Scottish HPV Archive as a Research Tissue Bank (REC Ref 11/AL/0174) for HPV related research on archive samples. Samples are available for the present project though application to the Archive Steering Committee (HPV Archive Application Ref 0034). RNA and protein were extracted from the samples using Trizol as described by the manufacturer (ThermoFischer Scientific, USA) and analysed as described.

\section{Tissue microarray and immunohistochemistry}

A cervical cancer tissue microarray (TMA) containing 39 cases of cervical cancer and 9 cases of normal cervical tissue (in duplicate) were purchased from GeneTex, Inc. (GTX21468). Slides were deparaffinised in xylene, rehydrated in a graded series of ethanol solutions and subjected to antigen retrieval in citric acid. Slides were blocked in normal serum and incubated in primary antibody (USP13 (D4P3M; 12577, Cell signalling Technologies (CST)) or Mcl-1 (S-19; sc-819, Santa Cruz Biotechnology (SCBT)) overnight at $4{ }^{\circ} \mathrm{C}$. Slides were then processed using the VECTASTAIN $^{\circledR}$ Universal Quick HRP Kit (PK7800; Vector Laboratories) as per the manufacturer's instructions. Immunostaining was visualised using 3,3'-diaminobenzidine (Vector ${ }^{\circledR}$ DAB (SK-4100; Vector Laboratories)). USP13 and Mcl-1 immunostaining quantification was automated using ImageJ with the IHC Profiler plug-in [75]. Histology scores (H-score) were calculated based on the percentage of positively stained tumour cells and the staining intensity grade [76]. The staining intensities were classified into the following four categories: 0, no staining; 1, low positive staining; 2 , positive staining; 3 , strong positive staining. $\mathrm{H}$ score was calculated by the following formula: ( $3 \mathrm{x}$ percentage of strong positive tissue $)+(2 \mathrm{x}$ percentage of positive tissue) + (percentage of low positive tissue), giving a range of 0 to 300 .

\section{Cell culture}

HeLa (HPV18 + cervical adenocarcinoma cells), SW756 (HPV18 + cervical squamous carcinoma cells), $\mathrm{SiHa}$ (HPV16 + cervical squamous carcinoma cells), CaSKi (HPV16 + cervical squamous carcinoma cells) and C33A (HPV- cervical squamous carcinoma) cells obtained from the ATCC and grown in DMEM supplemented with $10 \%$ FBS (ThermoFisher Scientific, USA) and $50 \mathrm{U} / \mathrm{mL}$ penicillin. Primary normal human keratinocytes (NHKs) were maintained as in [77]. Cells were negative for Mycoplasma during this investigation. Cell identify was confirmed by STR profiling.

\section{Plasmids, siRNA and inhibitors}

pRK5-FLAG-USP13 (Addgene plasmid \#61741) and pcDNA3.1-V5-hMcl-1 (Addgene plasmid \#25375) were purchased from Addgene (Cambridge, MA, USA). The USP13 C345A mutant was created using the Q5 SiteDirected Mutagenesis Kit (New England BioLabs Inc, MA, USA) using the following primers: $F-5^{\prime}$ TGGGCAA CAGCGCCTATCTCAGCTC ' 3 and $\mathrm{R}-$ 5' $^{\prime}$ GGTTCTT CAGACCCGTGT '3. HA-Ubiquitin, HA-Ubiquitin (K48R) and HA-Ubiquitin (K63R) were a kind gift from Prof. Paul Lehner (University of Cambridge, UK). siRNA targeting USP13 and JUN were purchased from Qiagen (FlexiTube GeneSolution GS8975 for USP13; SI03074498, SI03061968, 632 SI00058100, SI00058093; FlexiTube GeneSolution GS3725 for JUN; SI03077599, SI00034678, SI00034671, SI00034664). The small molecule inhibitors MG132, JNK-IN-8, Spautin-1 and ABT-263 were purchased from Calbiochem and used at the indicated concentrations.

\section{Transfections and mammalian cell lysis}

Transfection of plasmid DNA or siRNA was performed with a DNA to Lipofectamine ${ }^{\circledR} 2000$ (ThermoFisher) ratio of $1: 2.5$. 48 or $72 \mathrm{~h}$ post transfection, cells were lysed in lysis buffer for western blot analysis, or reseeded into new plates for growth curve analysis, colony formation assays or soft agar assays.

\section{Deubiquitination assays}

FLAG-USP13, V5-hMcl-1 and/or HA-ubiquitin plasmids were transfected into HEK293 cells using Lipofectamine 
2000. After $40 \mathrm{~h}$, the cells were treated with $20 \mu \mathrm{M}$ MG132 (Calbiochem) for $8 \mathrm{~h}$. The cells were then washed with PBS and lysed in HEPES buffer supplemented with $100 \mu \mathrm{M} \mathrm{N}$ ethylmaleimide and protease inhibitor cocktail (Roche). The lysates were centrifuged and incubated with $2 \mu \mathrm{g}$ Mcl-1 antibody (S-19; sc-819, SCBT) at $4{ }^{\circ} \mathrm{C}$ overnight with continuous rotation. A/G agarose beads were then added, and the lysate/antibody/bead mix was incubated at $4{ }^{\circ} \mathrm{C}$ overnight with continuous rotation. Beads were then washed in HEPES buffer and boiled in Laemmli loading buffer prior to SDS PAGE and western blot analysis with an anti-HA antibody (HA-7; H9658, Sigma).

\section{Immunoprecipitations}

Assays were performed as previously described [78]. Briefly, cells were transfected with FLAG-USP13, V5hMcl-1 or both. Cell lysates were harvested and then incubated with USP13, FLAG or Mcl-1 antibodies and incubated at $4{ }^{\circ} \mathrm{C}$ overnight with continuous rotation. A/G agarose beads were then added, and the lysate/antibody/ bead mix was incubated at $4{ }^{\circ} \mathrm{C}$ overnight with continuous rotation. Beads were then washed in lysis buffer and boiled in Laemmli loading buffer prior to SDS PAGE and western blot analysis.

\section{Western blot analysis}

Equal amounts of protein from cell lysates were separated by SDS PAGE and transferred onto a nitrocellulose membrane by a semi-dry transfer method (Trans Blot ${ }^{\circ}$ SD SemiDry Transfer cell, Bio-Rad, USA). Membranes were blocked with $5 \%$ milk solution before incubation with primary antibodies at 1:1000 dilution unless otherwise stated: USP13 (D4P3M; 12577, CST), Mcl-1 (S-19; sc-819, SCBT), Phospho-c-Jun (Ser73) (D47G9; 3270, CST), c-Jun (60A8; 9165, CST), FLAG (F1804, Sigma-Aldrich), HA (HA-7; H9658, Sigma) and GAPDH (SCBT; sc365062) (1:5000) as a loading control. Horseradish peroxidase (HRP)-conjugated secondary antibodies (Sigma Aldrich, USA) were used at a 1:5000 dilution. Proteins were detected using WesternBright ECL (Advansta, USA) and visualised on X-ray film.

\section{Chromatin immunoprecipitation}

Putative AP-1 binding sites in the USP13 3'UTR were identified using the AceView program. HeLa cells were treated with JNK-IN-8 for the required incubation time. Cells were processed for ChIP analysis as previously described [79]. Briefly, cells were fixed in $1 \%$ formaldehyde for $10 \mathrm{~min}$ at room temperature, quenched in $0.25 \mathrm{M}$ glycine, and washed in ice-cold PBS. Cells were harvested by scraping and then lysed in cell lysis buffer $(10 \mathrm{mM}$ Tris-HCl, $\mathrm{pH} 8.0,10 \mathrm{mM} \mathrm{NaCl}, 0.2 \% \mathrm{NP}-40,10 \mathrm{mM}$ sodium butyrate, $50 \mu \mathrm{g} / \mathrm{ml}$ phenylmethylsulfonyl fluoride (PMSF), $1 \times$ complete protease inhibitor). Nuclei were collected by centrifugation at $2,500 \mathrm{rpm}$ at $4{ }^{\circ} \mathrm{C}$ and resuspended in nuclear lysis buffer (50 mM Tris-HCl, pH 8.1, $10 \mathrm{mM}$ EDTA, $1 \%$ SDS, $10 \mathrm{mM}$ sodium butyrate, $50 \mu \mathrm{g} / \mathrm{ml}$ PMSF, $1 \times$ complete protease inhibitor). Extracted chromatin was then sonicated and chromatin concentration was determined. Approximately $100 \mu \mathrm{g}$ of chromatin from each sample was used for the experiment. c-Jun was immunoprecipitated using a ChIP grade anti-c-Jun antibody (60A8; 9165, CST). A/G magnetic beads were used to pull down the antibody-chromatin complex. To show antibody specificity, each of the samples were pulled down with an IgG isotype control. The immunoprecipitated chromatin was then processed for quantitative PCR (qPCR); the primer sequences used are available on request. Fold-enrichment compared to negative control $\operatorname{IgG}$ isotype control was calculated as in [80].

\section{RNA extraction, CDNA synthesis and quantitative Real Time-PCR}

RNA extraction for qRT-PCR was performed using an E.Z. N.A Total RNA Kit I (Omega bio-tek, USA). cDNA was synthesised with $1 \mu \mathrm{g}$ of input RNA and iScript cDNA synthesis kit (Bio Rad, USA). qRT-PCR was performed on the synthesised cDNA on a Corbett Rotor-Gene 6000 using QuantiFast SYBR Green PCR kit (Qiagen, USA) and analysed using the $\Delta \Delta C T$ method [75] normalised to the U6 housekeeping gene. The following primer were used: USP13 Forward 5'-GCGAAATCAGGCTATTCAGG-3', Reverse 5'-TTGTAAATCACCCATCTTCCTTCC-3'; $M C L$ 1 Forward 5'-AAAGCCTGTCTGCCAAAT- $3^{\prime}$, Reverse $5^{\prime}$ CCTATAAACCCACCACTC-3'; JUN Forward 5'-TTCT ATGACGATGCCCTCAACGC-3', Reverse 5'-GCTCTG TTTCAGGATCTTGGGGTTAC- $3^{\prime}$ and $U 6$ Forward 5'-CTCGCTTCGGCAGCACA-3', Reverse 5'-AACGCTT CACGAATTTGCGT-3'.

\section{Cell viability (MTT) assays}

Cells were seeded in triplicate in 96-well plates and subjected to the indicated treatments for $48 \mathrm{~h}$ before adding MTT reagent and measuring the absorbance at $570 \mathrm{~nm}$ according to the manufacturer's instructions (Sigma, USA). For combination matrices, cells were seeded in 96-well plates. After $24 \mathrm{~h}$, cells were treated with ABT-263 (dose range of $0-5 \mu \mathrm{M})$ and Spautin-1 (dose range of $0-20 \mu \mathrm{M}$ ) in a $6 \times 6$ matrix. Cells were cultured with inhibitors for $48 \mathrm{~h}$ and cell viability was determined using MTT assay as above. The Bliss synergy heatmap and synergy score was calculated using SynergyFinder [81]. 


\section{Colony formation assay}

48 or $72 \mathrm{~h}$ post treatment or transfection, cells were trypsinised and reseeded in a six well plate at 500 cells per well and left to incubate for 14-21 days. Colonies were then stained (1\% crystal violet, $25 \%$ methanol) and colonies were counted manually. Each experiment was repeated a minimum of 3 times.

\section{Soft agar assay}

Cells were treated or transfected as required. $60 \mathrm{~mm}$ dishes were coated with a layer of $1 \%$ agarose (ThermoFisher Scientific) in 2X DMEM (ThermoFisher Scientific) supplemented with $20 \%$ FBS. 48 or $72 \mathrm{~h}$ post transfection, cells were trypsinised and resuspended in $0.7 \%$ agarose in $2 \mathrm{X}$ DMEM (ThermoFisher Scientific) supplemented with $20 \%$ FBS at 1000 cells $/ \mathrm{mL}$. Once set, DMEM supplemented with $10 \%$ FBS and $50 \mathrm{U} / \mathrm{mL}$ penicillin was added. The plates were then incubated for 14-21 days. Each experiment was repeated at least three times. Visible colonies were counted manually.

\section{Annexin V assay}

Annexin V apoptosis assay (TACS Annexin V kit; 4830$250-\mathrm{K}$ ) was performed as indicated on the product datasheet. Briefly, cells seeded in 6 well plates were treated or transfected as required. Cells were trypsinised and collected by centrifugation. $1 \times 10^{6}$ cells were then incubated in $100 \mu \mathrm{L}$ Annexin $\mathrm{V}$ reagent $(10 \mu \mathrm{L} 10 \mathrm{x}$ binding buffer, $10 \mu \mathrm{L}$ propidium iodide, $1 \mu \mathrm{L}$ Annexin V-FITC (diluted 1 in

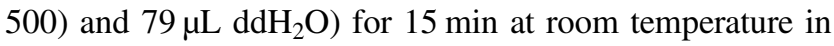
the dark. Samples were diluted in $1 \times$ binding buffer before analysis by flow cytometry. Samples were processed on a CytoFLEX S flow cytometer (Beckman Coulter) and analysed using CytExpert (Beckman Coulter).

\section{Statistical analysis}

Data points from at least three individual, biological repeats is shown in each graph. Unless otherwise indicated in the figure legends, data was analysed using a two-tailed, unpaired Student's $t$-test and graphs were prepared using the GraphPad Prism software (GraphPad, USA). Correlation was determined using Spearman's analysis. Error bars represent the mean $+/-$ standard deviation. Statistical significance was determined as follows: $* P<0.05, * * P<0.01$, $* * * P<0.001$

Acknowledgements We are particularly grateful to Prof. Roger Davis (UMASS, United States) and Dr Yihong Ye (NIDDK, NIH, USA) for generous provision of reagents through the Addgene repository. We thank Prof. Paul Lehner (University of Cambridge) for the ubiquitin expression plasmids and Prof. Adrian Whitehouse (University of Leeds) for providing the V5 antibody. We additionally thank the Scottish HPV Investigators Network (SHINE) for providing HPV positive cytology samples. Finally, we thank Dr Stephen Griffin for critical reading of the manuscript.

Funding This work was supported by the Wellcome Institutional Strategic Support Fund (ISSF) funding to ELM (204825/Z/16/Z) and Medical Research Council (MRC) funding to AM (MR/ K012665 and $\mathrm{MR} / \mathrm{S} 001697 / 1)$. MRP is funded by Biotechnology and Biological Sciences Research Council studentship (BB/M011151/1). JAS is funded by a Faculty of Biological Sciences, University of Leeds scholarship. The funders had no role in study design, data collection and analysis, decision to publish, or preparation of the manuscript.

\section{Compliance with ethical standards}

Conflict of interest The author declares no competing interests.

Publisher's note Springer Nature remains neutral with regard to jurisdictional claims in published maps and institutional affiliations.

Open Access This article is licensed under a Creative Commons Attribution 4.0 International License, which permits use, sharing, adaptation, distribution and reproduction in any medium or format, as long as you give appropriate credit to the original author(s) and the source, provide a link to the Creative Commons license, and indicate if changes were made. The images or other third party material in this article are included in the article's Creative Commons license, unless indicated otherwise in a credit line to the material. If material is not included in the article's Creative Commons license and your intended use is not permitted by statutory regulation or exceeds the permitted use, you will need to obtain permission directly from the copyright holder. To view a copy of this license, visit http://creativecommons. org/licenses/by/4.0/.

\section{References}

1. Clague MJ, Urbé S. Ubiquitin: same molecule, different degradation pathways. Cell. 2010;143:682-5.

2. Swatek KN, Komander D. Ubiquitin modifications. Cell Res. 2016;26:399-422.

3. Akutsu M, Dikic I, Bremm A. Ubiquitin chain diversity at a glance. J Cell Sci. 2016;129:875-80.

4. Huang X, Dixit VM. Drugging the undruggables: exploring the ubiquitin system for drug development. Cell Res 2016;26:484-98.

5. Komander D, Clague MJ, Urbé S. Breaking the chains: structure and function of the deubiquitinases. Nat Rev Mol Cell Biol. 2009;10:550-63.

6. Alwan HAJ, Leeuwen JEM. van. UBPY-mediated epidermal growth factor receptor (EGFR) de-ubiquitination promotes EGFR degradation. J Biol Chem. 2007;282:1658-69.

7. Yuan J, Luo K, Zhang L, Cheville JC, Lou Z. USP10 regulates p53 localization and stability by deubiquitinating p53. Cell. 2010;140:384-96.

8. Sun X-X, He X, Yin L, Komada M, Sears RC, Dai M-S. The nucleolar ubiquitin-specific protease USP36 deubiquitinates and stabilizes c-Myc. Proc Natl Acad Sci USA. 2015;112:3734-9.

9. Park MK, Yao Y, Xia W, Setijono SR, Kim JH, Vila IK, et al. PTEN self-regulates through USP11 via the PI3K-FOXO pathway to stabilize tumor suppression. Nat Commun. 2019;10:636-17. 
10. Li M, Brooks CL, Kon N, Gu W. A dynamic role of HAUSP in the p53-Mdm2 pathway. Mol Cell 2004;13:879-86.

11. Turnbull AP, Ioannidis S, Krajewski WW, Pinto-Fernandez A, Heride C, Martin ACL, et al. Molecular basis of USP7 inhibition by selective small-molecule inhibitors. Nature. 2017;550:481-6.

12. Crosbie EJ, Einstein MH, Franceschi S, Kitchener HC. Human papillomavirus and cervical cancer. Lancet (Lond, Engl) 2013;382 (Sep):889-99.

13. Hausen H. zur. Papillomaviruses and cancer: from basic studies to clinical application. Nat Rev Cancer. 2002;2:342-50.

14. Moody CA, Laimins LA. Human papillomavirus oncoproteins: pathways to transformation. Nat Rev Cancer. 2010;10:550-60.

15. Mller M, Prescott EL, Wasson CW, Macdonald A. Human papillomavirus E5 oncoprotein: function and potential target for antiviral therapeutics. Future Virol. 2015;10:27-39.

16. Spangle JM, Münger K. The HPV16 E6 oncoprotein causes prolonged receptor protein tyrosine kinase signaling and enhances internalization of phosphorylated receptor species. PLoS Pathog. 2013;9:e1003237.

17. He C, Mao D, Hua G, Lv X, Chen X, Angeletti PC, et al. The Hippo/YAP pathway interacts with EGFR signaling and HPV oncoproteins to regulate cervical cancer progression. EMBO Mol Med. 2015;7:1426-49.

18. Morgan EL, Macdonald A. Autocrine STAT3 activation in HPV positive cervical cancer through a virus-driven Rac1-NFKB-IL6 signalling axis. PLoS Pathog. 2019;15:e1007835.

19. Morgan EL, Macdonald A. JAK2 inhibition impairs proliferation and sensitises cervical cancer cells to cisplatin-induced cell death. Cancers. 2019;11:1934.

20. Morgan EL, Patterson MR, Ryder EL, Lee SY, Wasson CW, Harper KL, et al. MicroRNA-18a targeting of the STK4/MST1 tumour suppressor is necessary for transformation in HPV positive cervical cancer. PLoS Pathog. 2020;16:e1008624.

21. Bello JOM, Nieva LO, Paredes AC, Gonzalez AMF, Zavaleta LR, Lizano M. Regulation of the Wnt/ $\beta$-catenin signaling pathway by human papillomavirus E6 and E7 oncoproteins. Viruses. 2015;7:4734-55.

22. Scheffner M, Huibregtse JM, Vierstra RD, Howley PM. The HPV-16 E6 and E6-AP complex functions as a ubiquitin-protein ligase in the ubiquitination of p53. Cell. 1993;75:495-505.

23. Boyer SN, Wazer DE, Band V. E7 protein of human papilloma virus-16 induces degradation of retinoblastoma protein through the ubiquitin-proteasome pathway. Cancer Res. 1996;56:4620-4.

24. Huh K, Zhou X, Hayakawa H, Cho J-Y, Libermann TA, Jin J, et al. Human papillomavirus type 16 E7 oncoprotein associates with the cullin 2 ubiquitin ligase complex, which contributes to degradation of the retinoblastoma tumor suppressor. J Virol. 2007;81:9737-47.

25. Pim D, Collins M, Banks L. Human papillomavirus type 16 E5 gene stimulates the transforming activity of the epidermal growth factor receptor. Oncogene. 1992;7:27-32.

26. Straight SW, Hinkle PM, Jewers RJ, McCance DJ. The E5 oncoprotein of human papillomavirus type 16 transforms fibroblasts and effects the downregulation of the epidermal growth factor receptor in keratinocytes. J Virol. 1993;67:4521-32.

27. Wasson CW, Morgan EL, Müller M, Ross RL, Hartley M, Roberts S, et al. Human papillomavirus type 18 E5 oncogene supports cell cycle progression and impairs epithelial differentiation by modulating growth factor receptor signalling during the virus life cycle. Oncotarget. 2017;8:103581-600.

28. Wetherill LF, Wasson CW, Swinscoe G, Kealy D, Foster R, Griffin S, et al. Alkyl-imino sugars inhibit the pro-oncogenic ion channel function of human papillomavirus (HPV) E5. Antivir Res. 2018;158:113-21.

29. Zhang B, Srirangam A, Potter DA, Roman A. HPV16 E5 protein disrupts the c-Cbl-EGFR interaction and EGFR ubiquitination in human foreskin keratinocytes. Oncogene. 2005;24:2585-8.
30. An J, Mo D, Liu H, Veena MS, Srivatsan ES, Massoumi R, et al. Inactivation of the CYLD deubiquitinase by HPV E6 mediates hypoxia-induced NF-kappaB activation. Cancer Cell. 2008;14:394-407.

31. Karim R, Tummers B, Meyers C, Biryukov JL, Alam S, Backendorf $\mathrm{C}$, et al. Human papillomavirus (HPV) upregulates the cellular deubiquitinase UCHL1 to suppress the keratinocyte's innate immune response. PLoS Pathog. 2013;9:e1003384.

32. Lehoux M, Gagnon D, Archambault J. E1-mediated recruitment of a UAF1-USP deubiquitinase complex facilitates human papillomavirus DNA replication. J Virol. 2014;88:8545-55.

33. Kiran S, Dar A, Singh SK, Lee KY, Dutta A. The deubiquitinase USP46 is essential for proliferation and tumor growth of HPVtransformed cancers. Mol Cell. 2018;72:823-.e5.

34. Network CGAR, Medicine AEC of, Services AB, Hospital BC, Medicine $\mathrm{BC}$ of, Hope BRI of $\mathrm{C}$ of, et al. Integrated genomic and molecular characterization of cervical cancer. Nature. 2017;543:378-84.

35. Ge Z, Leighton JS, Wang Y, Peng X, Chen Z, Chen H, et al. Integrated genomic analysis of the ubiquitin pathway across cancer types. Cell Rep. 2018;23:213-e3.

36. Bansal N, Wright JD, Cohen CJ, Herzog TJ. Natural history of established low grade cervical intraepithelial (CIN 1) lesions. Anticancer Res. 2008;28:1763-6.

37. Chung YW, Jeong D, Won JY, Choi E-J, Choi YH, Kim IY. H(2) $\mathrm{O}(2)$-induced AP-1 activation and its effect on p21(WAF1/CIP1)mediated G2/M arrest in a p53-deficient human lung cancer cell. Biochemical biophysical Res Commun. 2002;293:1248-53.

38. Rio R, del, Noubade R, Subramanian M, Saligrama N, Diehl S, Rincon M, et al. SNPs upstream of the minimal promoter control IL2 expression and are candidates for the autoimmune diseasesusceptibility locus Aod2/Idd3/Eae3. Genes Immun. 2008;9:115-21.

39. Gupta S, Barrett T, Whitmarsh AJ, Cavanagh J, Sluss HK, Dérijard B, et al. Selective interaction of JNK protein kinase isoforms with transcription factors. EMBO J. 1996;15:2760-70.

40. Zhao X, Fiske B, Kawakami A, Li J, Fisher DE. Regulation of MITF stability by the USP13 deubiquitinase. Nat Commun. 2011;2:414-8.

41. Zhang J, Zhang P, Wei Y, Piao H-L, Wang W, Maddika S, et al. Deubiquitylation and stabilization of PTEN by USP13. Nat Cell Biol. 2013;15:1486-94.

42. Han C, Yang L, Choi HH, Baddour J, Achreja A, Liu Y, et al. Amplification of USP13 drives ovarian cancer metabolism. Nat Commun. 2016;7:13525-16.

43. Zhang S, Zhang M, Jing Y, Yin X, Ma P, Zhang Z, et al. Deubiquitinase USP13 dictates MCL1 stability and sensitivity to BH3 mimetic inhibitors. Nat Commun. 2018;9:215-12.

44. Zhang T, Zhao C, Luo L, Zhao H, Cheng J, Xu F. The expression of Mcl-1 in human cervical cancer and its clinical significance. Med Oncol. 2012;29:1985-91.

45. Lee W-S, Park Y-L, Kim N, Oh H-H, Son D-J, Kim M-Y, et al. Myeloid cell leukemia-1 regulates the cell growth and predicts prognosis in gastric cancer. Int J Oncol. 2015;46:2154-62.

46. Crawford RA, Caldwell C, Iles RK, Lowe D, Shepherd JH, Chard T. Prognostic significance of the bcl-2 apoptotic family of proteins in primary and recurrent cervical cancer. Br J Cancer. 1998;78:210-4.

47. Rahman SFA, Muniandy K, Soo YK, Tiew EYH, Tan KX, Bates TE, et al. Co-inhibition of BCL-XL and MCL-1 with selective BCL-2 family inhibitors enhances cytotoxicity of cervical cancer cell lines. Biochem Biophysics Rep. 2020;22:100756.

48. Liu J, Xia H, Kim M, Xu L, Li Y, Zhang L, et al. Beclin1 controls the levels of $\mathrm{p} 53$ by regulating the deubiquitination activity of USP10 and USP13. Cell. 2011;147:223-34.

49. Lian BSX, Yek AEH, Shuvas H, Rahman SFA, Muniandy K, Mohana-Kumaran N. Synergistic anti-proliferative effects of combination of ABT-263 and MCL-1 selective inhibitor A-1210477 on cervical cancer cell lines. BMC Res Notes 2018;11:197-7. 
50. Leverson JD, Phillips DC, Mitten MJ, Boghaert ER, Diaz D, Tahir SK, et al. Exploiting selective BCL-2 family inhibitors to dissect cell survival dependencies and define improved strategies for cancer therapy. Sci Transl Med. 2015;7:279ra40-279ra40.

51. Liu J, Shaik S, Dai X, Wu Q, Zhou X, Wang Z, et al. Targeting the ubiquitin pathway for cancer treatment. Biochimica et biophysica acta. 2015;1855:50-60.

52. Xu GW, Ali M, Wood TE, Wong D, Maclean N, Wang X, et al. The ubiquitin-activating enzyme $\mathrm{E} 1$ as a therapeutic target for the treatment of leukemia and multiple myeloma. Blood. 2010;115:2251-9.

53. Chen C, Meng Y, Wang L, Wang H-X, Tian C, Pang G-D, et al. Ubiquitin-activating enzyme E1 inhibitor PYR41 attenuates angiotensin II-induced activation of dendritic cells via the IкBa/ NF- $\mathrm{KB}$ and MKP1/ERK/STAT1 pathways. Immunology. 2014;142:307-19.

54. McHugh A, Fernandes K, South AP, Mellerio JE, Salas-Alanís JC, Proby CM, et al. Preclinical comparison of proteasome and ubiquitin E1 enzyme inhibitors in cutaneous squamous cell carcinoma: the identification of mechanisms of differential sensitivity. Oncotarget. 2018;9:20265-81.

55. Barghout SH, Patel PS, Wang X, Xu GW, Kavanagh S, Halgas O, et al. Preclinical evaluation of the selective small-molecule UBA1 inhibitor, TAK-243, in acute myeloid leukemia. Leukemia. 2019;33:37-51.

56. Hodge CD, Edwards RA, Markin CJ, McDonald D, Pulvino M, Huen MSY, et al. Covalent Inhibition of Ubc13 Affects Ubiquitin Signaling and Reveals Active Site Elements Important for Targeting. ACS Chem Biol. 2015;10:1718-28.

57. Fields AP, Justilien V, Murray NR. The chromosome $3 \mathrm{q} 26$ OncCassette: A multigenic driver of human cancer. Adv Biol Regul. 2016;60:47-63.

58. Wang Y, Ou Z, Sun Y, Yeh S, Wang X, Long J, et al. Androgen receptor promotes melanoma metastasis via altering the miRNA539-3p/USP13/MITF/AXL signals. Oncogene. 2017;36:1644-54.

59. Fang X, Zhou W, Wu Q, Huang Z, Shi Y, Yang K, et al. Deubiquitinase USP13 maintains glioblastoma stem cells by antagonizing FBXL14-mediated Myc ubiquitination. J Exp Med. 2017;214:245-67.

60. Ashkenazi A, Fairbrother WJ, Leverson JD, Souers AJ. From basic apoptosis discoveries to advanced selective BCL-2 family inhibitors. Nat Rev Drug Discov. 2017;16:273-84.

61. Blasio AD, Vento R, Fiore RD. Mcl-1 targeting could be an intriguing perspective to cure cancer. J Cell Physiol. 2018;233:8482-98.

62. Wei AH, Roberts AW, Spencer A, Rosenberg AS, Siegel D, Walter RB, et al. Targeting MCL-1 in hematologic malignancies: Rationale and progress. Blood Rev. 2020;44:100672.

63. Senichkin VV, Streletskaia AY, Gorbunova AS, Zhivotovsky B, Kopeina GS. Saga of Mcl-1: regulation from transcription to degradation. Cell death Differ. 2020;27:405-19.

64. Wu L, Lin Y, Feng J, Qi Y, Wang X, Lin Q, et al. The deubiquitinating enzyme OTUD1 antagonizes BH3-mimetic inhibitor induced cell death through regulating the stability of the MCL1 protein. Cancer Cell Int. 2019;19:222-11.

65. Wu X, Luo Q, Zhao P, Chang W, Wang Y, Shu T, et al. JOSD1 inhibits mitochondrial apoptotic signalling to drive acquired chemoresistance in gynaecological cancer by stabilizing MCL1. Cell Death Differ. 2020;27:55-70.
66. Oltersdorf T, Elmore SW, Shoemaker AR, Armstrong RC, Augeri DJ, Belli BA, et al. An inhibitor of Bcl-2 family proteins induces regression of solid tumours. Nature. 2005;435:677-81.

67. Tse C, Shoemaker AR, Adickes J, Anderson MG, Chen J, Jin S, et al. ABT-263: a potent and orally bioavailable Bcl-2 family inhibitor. Cancer Res. 2008;68:3421-8.

68. Roberts AW, Davids MS, Pagel JM, Kahl BS, Puvvada SD, Gerecitano JF, et al. Targeting BCL2 with venetoclax in relapsed chronic lymphocytic leukemia. New Engl J Med. 2016;374:311-22.

69. Mazumder S, Choudhary GS, Al-Harbi S, Almasan A. Mcl-1 Phosphorylation defines ABT-737 resistance that can be overcome by increased NOXA expression in leukemic B cells. Cancer Res. 2012;72:3069-79.

70. Wang B, Ni Z, Dai X, Qin L, Li X, Xu L, et al. The Bcl-2/xL inhibitor ABT-263 increases the stability of Mcl-1 mRNA and protein in hepatocellular carcinoma cells. Mol Cancer. 2014;13:98-11.

71. Mukherjee N, Skees J, Todd KJ, West DA, Lambert KA, Robinson WA, et al. MCL1 inhibitors S63845/MIK665 plus Navitoclax synergistically kill difficult-to-treat melanoma cells. Cell Death Dis. 2020;11:443-14.

72. Chen S, Dai Y, Pei X-Y, Myers J, Wang L, Kramer LB, et al. $\mathrm{CDK}$ inhibitors upregulate $\mathrm{BH} 3$-only proteins to sensitize human myeloma cells to $\mathrm{BH} 3$ mimetic therapies. Cancer Res. 2012;72:4225-37.

73. Kiprianova I, Remy J, Milosch N, Mohrenz IV, Seifert V, Aigner A. et al. Sorafenib sensitizes glioma cells to the BH3 mimetic ABT-737 by targeting MCL1 in a STAT3-dependent manner. Neoplasia (N. Y, NY). 2015;17.:564-73.

74. Collaboration $\mathrm{C}$ for CCM-A. Reducing uncertainties about the effects of chemoradiotherapy for cervical cancer: a systematic review and meta-analysis of individual patient data from 18 randomized trials. J Clin Oncol. 2008;26:5802-12.

75. Varghese F, Bukhari AB, Malhotra R, De A. IHC Profiler: an open source plugin for the quantitative evaluation and automated scoring of immunohistochemistry images of human tissue samples. PloS One 2014;9:e96801.

76. Detre S, Jotti GS, Dowsett MA. "Quickscore" method for immunohistochemical semiquantitation: validation for oestrogen receptor in breast carcinomas. J Clin Pathol. 1995;48:876-8.

77. Morgan EL, Wasson CW, Hanson L, Kealy D, Pentland I, McGuire V, et al. STAT3 activation by E6 is essential for the differentiation-dependent HPV18 life cycle. PLoS Pathog. 2018;14:e1006975.

78. Griffiths DA, Abdul-Sada H, Knight LM, Jackson BR, Richards $\mathrm{K}$, Prescott EL, et al. Merkel cell polyomavirus small T antigen targets the NEMO adaptor protein to disrupt inflammatory signaling. J Virol. 2013;87:13853-67.

79. Das D, Bristol ML, Smith NW, James CD, Wang X, Pichierri P, et al. Werner helicase control of human papillomavirus 16 E1-E2 DNA replication is regulated by SIRT1 deacetylation. mBio 2019;10(Mar):260.

80. Pentland I, Campos-León K, Cotic M, Davies K-J, Wood CD, Groves IJ, et al. Disruption of CTCF-YY1-dependent looping of the human papillomavirus genome activates differentiation-induced viral oncogene transcription. PLoS Biol 2018;16:e2005752.

81. Ianevski A, Giri AK, Aittokallio T. SynergyFinder 2.0: visual analytics of multi-drug combination synergies. Nucleic Acids Res. 2020;48:W488-93. 